\title{
A cullin 4B-RING E3 ligase complex fine-tunes pancreatic $\delta$ cell paracrine interactions
}

\author{
Qing Li, ${ }^{1}$ Min Cui, ${ }^{1}$ Fan Yang, ${ }^{1}$ Na Li, ${ }^{1}$ Baichun Jiang, ${ }^{2}$ Zhen Yu, ${ }^{1}$ Daolai Zhang, ${ }^{3}$ Yijing Wang, ${ }^{3}$ Xibin Zhu, ${ }^{1}$ Huili Hu, ${ }^{2}$ Pei-Shan Li, ${ }^{2}$ \\ Shang-Lei Ning, ${ }^{3}$ Si Wang, ${ }^{1}$ Haibo Qi, ${ }^{1}$ Hechen Song, ${ }^{1}$ Dongfang He,,${ }^{1,3}$ Amy Lin, ${ }^{4}$ Jingjing Zhang, ${ }^{5}$ Feng Liu, ${ }^{5}$ Jiajun Zhao, ${ }^{6}$ Ling Cao, ${ }^{6}$ \\ Fan Yi, ${ }^{7}$ Tian Xue, ${ }^{8}$ Jin-Peng Sun, ${ }^{3,4}$ Yaoqin Cong, ${ }^{2}$ and Xiao $\mathrm{Yu}^{1}$

\begin{abstract}
1Key Laboratory Experimental Teratology of the Ministry of Education and Department of Physiology, ${ }^{2}$ Key Laboratory Experimental Teratology of the Ministry of Education and Department of Genetics, and ${ }^{3}$ Department of Biochemistry, Shandong University School of Medicine, Jinan, Shandong, China. ${ }^{4}$ Duke University, School of Medicine, Durham, North Carolina, USA. ${ }^{5}$ The Second Xiangya Hospital, Central South University, Changsha, Hunan, China. ${ }^{6}$ Department of Endocrinology, Shandong Provincial Hospital affiliated to Shandong University, Jinan, China. 'Department of Pharmacology, Shandong University School of Medicine, Jinan, Shandong, China. ${ }^{8}$ Hefei National Laboratory for Physical Science at Microscale, School of Life Science, University of Science and Technology of China, Hefei, Anhui, China.
\end{abstract}

\begin{abstract}
Somatostatin secreted by pancreatic $\delta$ cells mediates important paracrine interactions in Langerhans islets, including maintenance of glucose metabolism through the control of reciprocal insulin and glucagon secretion. Disruption of this circuit contributes to the development of diabetes. However, the precise mechanisms that control somatostatin secretion from islets remain elusive. Here, we found that a super-complex comprising the cullin 4B-RING E3 ligase (CRL4B) and polycomb repressive complex 2 (PRC2) epigenetically regulates somatostatin secretion in islets. Constitutive ablation of CUL4B, the core component of the CRL4B-PRC2 complex, in $\delta$ cells impaired glucose tolerance and decreased insulin secretion through enhanced somatostatin release. Moreover, mechanistic studies showed that the CRL4B-PRC2 complex, under the control of the $\delta$ cell-specific transcription factor hematopoietically expressed homeobox (HHEX), determines the levels of intracellular calcium and cAMP through histone posttranslational modifications, thereby altering expression of the Cav1.2 calcium channel and adenylyl cyclase 6 (AC6) and modulating somatostatin secretion. In response to high glucose levels or urocortin 3 (UCN3) stimulation, increased expression of cullin 4B (CUL4B) and the PRC2 subunit histone-lysine $\mathrm{N}$-methyltransferase EZH2 and reciprocal decreases in Cav1.2 and AC6 expression were found to regulate somatostatin secretion. Our results reveal an epigenetic regulatory mechanism of $\delta$ cell paracrine interactions in which CRL4B-PRC2 complexes, Cav1.2, and AC6 expression fine-tune somatostatin secretion and facilitate glucose homeostasis in pancreatic islets.
\end{abstract}

\section{Introduction}

The functions of different cell types within Langerhans islets are precisely regulated by paracrine interactions, and these coordinate the rate and level of reciprocal insulin and glucagon secretion, thereby maintaining glucose homeostasis in response to changes in nutrient levels in different physiological contexts (1-4). The disruption of this fine-tuned islet circuit by the aberrant regulation of paracrine islet interactions plays an important role in the development of diabetes (1-9). For example, the loss of the important paracrine factor urocortin 3 (UCN3) in pancreatic $\beta$ cells caused dysregulation of a somatostatin-mediated negative feedback loop between islet $\delta$ and $\beta$ cells, resulting in aberrant glucose-

Authorship note: Q. Li, M. Cui, and F. Yang contributed equally to this work. Y. Gong and X. Yu contributed equally to this work.

Note regarding evaluation of this manuscript: Manuscripts authored by scientists associated with Duke University, The University of North Carolina at Chapel Hill, Duke-NUS, and the Sanford-Burnham Medical Research Institute are handled not by members of the editorial board but rather by the science editors, who consult with selected external editors and reviewers.

Conflict of interest: The authors have declared that no conflict of interest exists.

Submitted: October 21, 2016; Accepted: April 20, 2017

Reference information: / Clin Invest. 2017;127(7):2631-2646.

https://doi.org/10.1172/JCI91348. induced insulin secretion and contributing to the pathophysiology of diabetes $(5,10,11)$. Recently, the functional importance of pancreatic $\delta$ cells in maintaining glucose homeostasis is increasingly being appreciated. More specifically, several studies have shown that somatostatin secretion from pancreatic $\delta$ cells is regulated by intracellular calcium and cyclic AMP, whereas somatostatin synthesis in pancreatic $\delta$ cells is regulated by the cAMP and $\beta$-arrestin 1 pathways $(12,13)$. In spite of this progress, whether an epigenetic regulatory mechanism underlies the crosstalk between pancreatic $\delta$ and $\beta$ cells has not been investigated.

Cullin family members accompanied by their partners in cullin-RING E3 ligase complexes (CRLs) play important roles in neuron activity, in cardiovascular function, and in the development of the reproductive system and pancreatic islets $(14,22)$. In particular, cullin 4B (CUL4B) mutations cause mental retardation, short stature, and obesity $(23,24)$. Unlike CUL4A and other cullins, CUL4B harbors an $\mathrm{N}$-terminal nuclear localization sequence that directs it to nucleus to regulate cell functions in an epigenetic manner (18, 25-27). Recently, we have shown that cullin 4B-RING E3 ligase (CRL4B) functions as a transcriptional corepressor by catalyzing H2AK119 ubiquitination (H2A119ub1) and coordinating with polycomb repressive complex 2 (PRC2) to regulate histone $\mathrm{H} 3$ lysine 27 (H3K27) trimethylation $(28,29)$. Other studies have demon- 
A

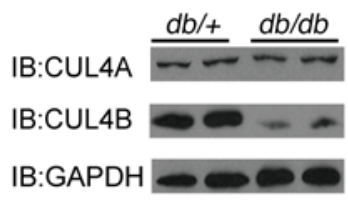

B

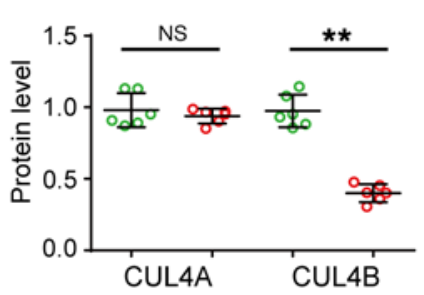

C
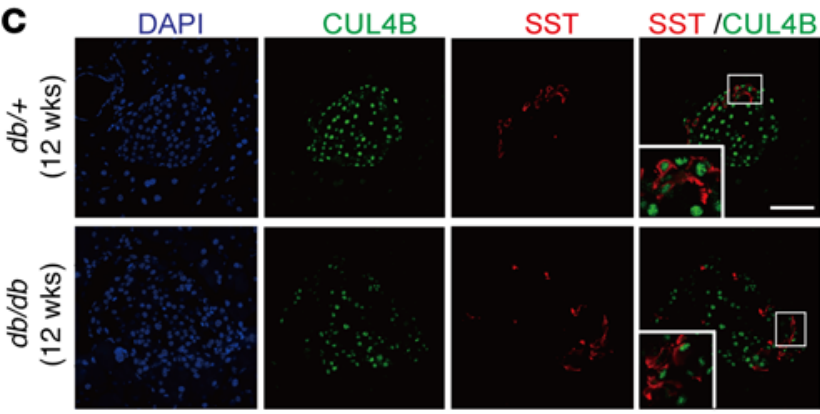

$\mathbf{E}$
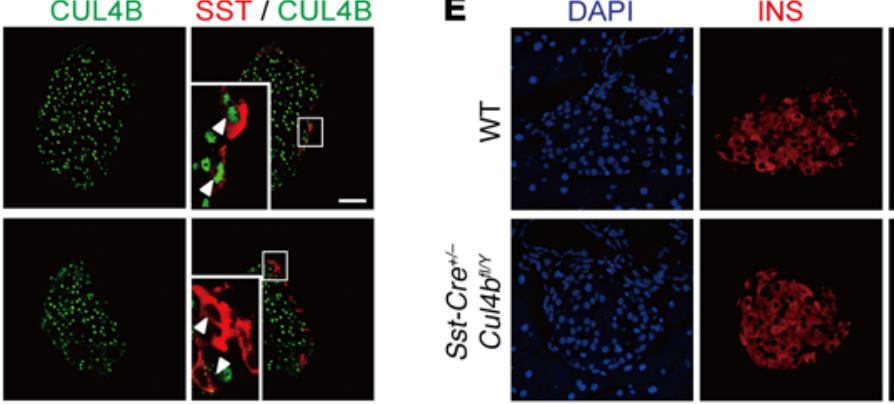

SST

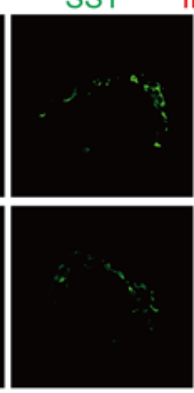

INS / SST/ DAPI

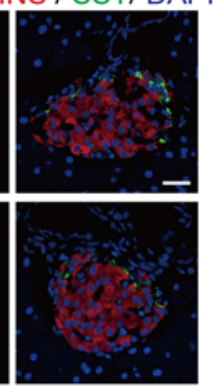

G
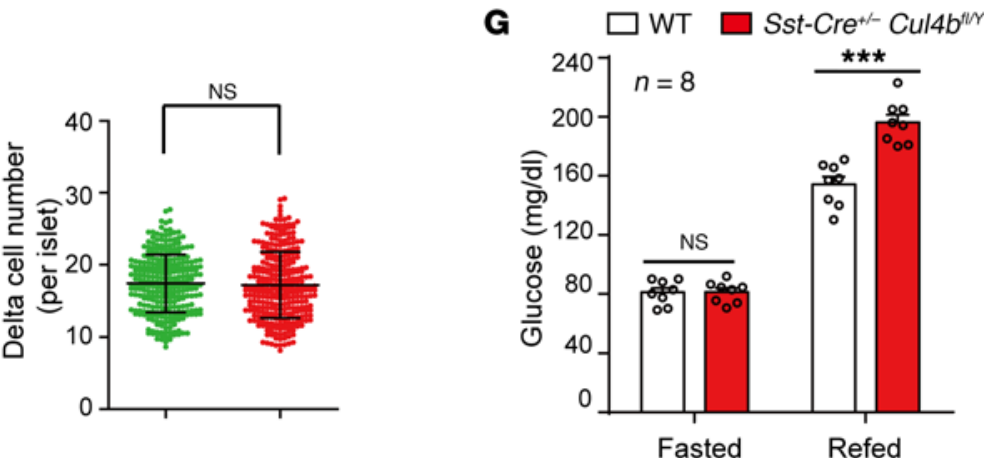
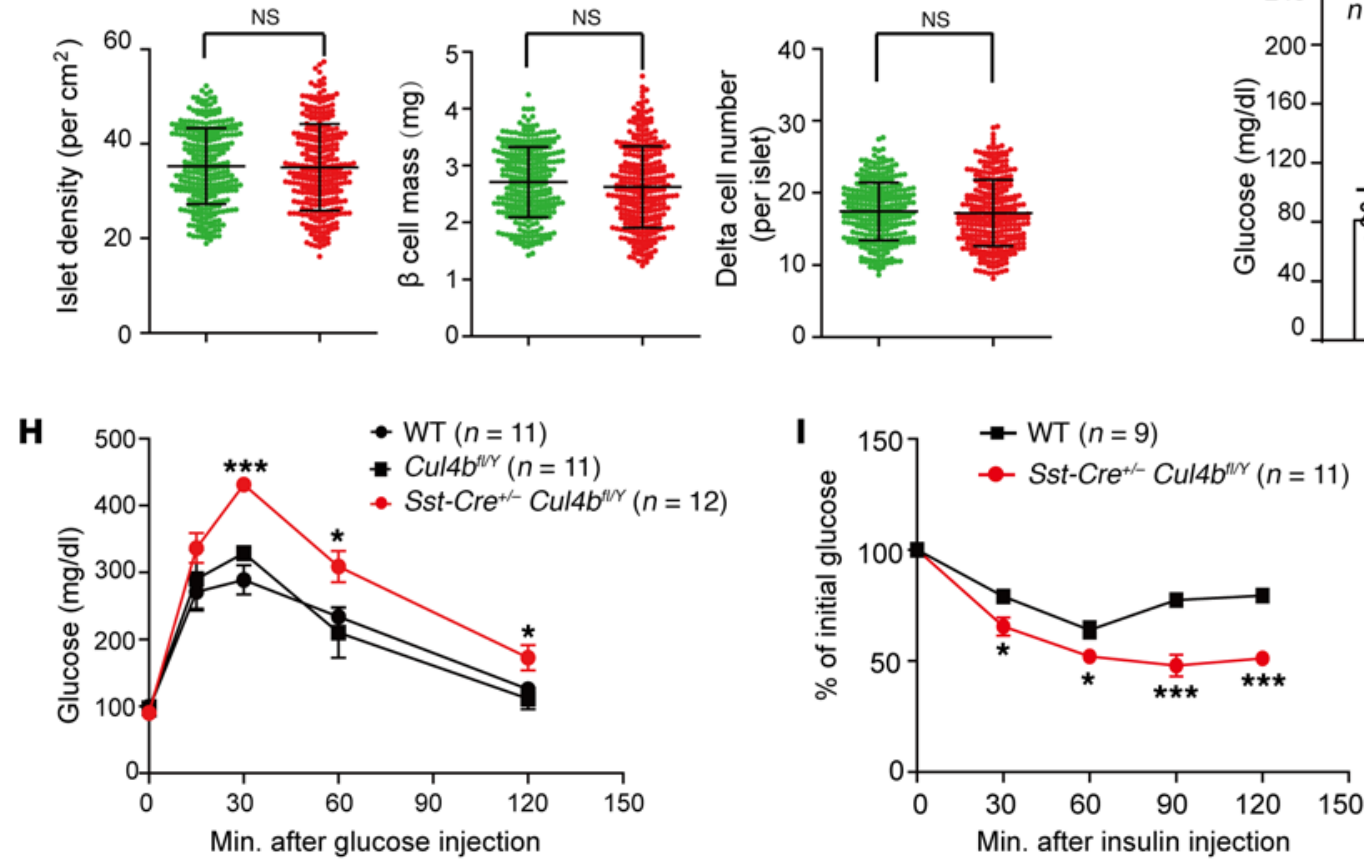

Figure 1. CUL4B deficiency in pancreatic $\delta$ cells impairs glucose metabolism. (A and B) Western blots and quantitative data for CUL4A and CUL4B protein levels in islets from 12-week-old diabetic $d b / d b$ mice and their heterozygous littermates $(d b /+) . n=6$ mice per group. Representative Western blots from at least 3 independent experiments are shown. (C) Immunostaining for CUL4B (green) and somatostatin (SST, red) in pancreatic sections from $d b / d b$ and $d b /+$ mice. Scale bar: $100 \mu \mathrm{m} . n=6$ mice per group; 4-7 random areas were selected from each islet section, and 10 sections were randomly selected from each mouse. (D) Confirmation of pancreatic $\delta$ cell-specific CUL4B deficiency (Sst-Cre ${ }^{+/-}$Cul4 $\left.b^{f / /}\right)$ through immunofluorescence. The colocalization of somatostatin (red) and CUL4B (green) in $\delta$ cells of WT mice was absent in Sst-Cre ${ }^{+/-}$Cul $4 b^{f / \gamma}$ mice. Scale bar: $100 \mu \mathrm{m}$. (E) Immunostaining for insulin (red) and somatostatin (green) in WT and Sst-Cre ${ }^{+/-}$Cul $4 b^{f / / \gamma}$ mice. Scale bar: $50 \mu \mathrm{m}$. (F) Quantitative data for islet density, pancreatic $\delta$ cell number, and $\beta$ cell mass. $n=6$ mice per group; 4-10 random areas were selected from each section, and 12 sections were randomly selected from each mouse. (G) The fasting and fed blood glucose levels of Sst-Cre ${ }^{+/-} C u l 4 b^{f / \gamma}$ mice and their WT littermates. $n=8$ mice per group. (H) Clucose tolerance test for Sst-Cre ${ }^{+/-}$Cul4b $b^{f / Y}$ mice and their WT littermates $(n=11-12)$. (I) Insulin tolerance test for Sst-Cre $e^{+/-}$Cul4b $b^{f / Y}$ mice and their WT littermates. Insulininduced decreases in blood glucose levels were significantly lower in Sst-Cre ${ }^{+/-}$Cul $4 b^{f / / \gamma}$ mice than in their WT littermates, and they did not return to baseline levels at the 2 -hour time point, whereas the levels of their WT littermates did $(n=9-11) .{ }^{*} P<0.05 ;{ }^{* *} P<0.01 ;{ }^{* *} P<0.001$. $d b / d b$ mice were compared with their $d b /+$ littermates, and Sst-Cre ${ }^{+/-} C u l 4 b^{f / / \gamma}$ mice were compared with their WT littermates. Error bars in $\mathbf{F}$ represent mean \pm SD; other bars represent mean \pm SEM. All data were analyzed using 1-way ANOVA. 
strated that CRL4 ubiquitinates WD repeat-containing protein 5 (WDR5), a core subunit of H3K4 methyltransferase complexes, for degradation in the nucleus, thereby promoting increased $\mathrm{H} 3 \mathrm{~K} 4$ methylation levels (30). However, whether CUL4B or its E3 ligase complex CRL4B-PRC2 participates in the functioning or development of Langerhans islets has not been studied.

In this study, we found that CUL4B expression levels are decreased in $d b / d b$ mice. Therefore, we crossed mice expressing Cre under the insulin II promotor (Ins2-Cre) and mice expressing Cre under the somatostatin promoter (Sst-Cre) with Cul4 $b^{f / f l}$ mice to characterize CUL4B functions in specific cell types of the islet circuit. Although Ins2-Cre Cul4 $b^{f / Y}$ male mice were found to exhibit normal plasma glucose levels, the constitutive ablation of CUL4B in pancreatic $\delta$ cells impaired glucose tolerance and reduced insulin secretion through enhanced somatostatin release. Moreover, mechanistic studies revealed that the CUL4B-PRC2 complex controls intracellular calcium and cAMP levels by epigenetically regulating the expression of ion channel CACNA1C (Cav1.2) and adenylyl cyclase 6 (AC6), thus adjusting somatostatin secretion from pancreatic $\delta$ cells.

\section{Results}

CUL4B ablation in pancreatic $\delta$ cells other than $\beta$ cells causes glucose intolerance. Previous studies have shown that mutations of $C U L 4 B$ in patients lead to obesity $(24,31)$. To understand the potential roles of CUL4B in glucose homeostasis, we examined protein levels of CUL4B in the pancreatic islets of obese diabetic $d b / d b$ mice. Total levels of islet CUL4B expression were decreased approximately 3-fold in 12-week-old $d b / d b$ mice compared with their littermate $d b /+$ controls, whereas the expression of its para$\log$ CUL4A remained unaltered (Figure 1, A and B; see complete unedited blots in the supplemental material; supplemental material available online with this article; https://doi.org/10.1172/ JCI91348DS1). Specifically, immunofluorescence results revealed that CUL4B expression decreased more drastically in pancreatic $\delta$ cells than in total islets in 12-week-old and 26-week-old $d b / d b$ mice (Figure $1 \mathrm{C}$ and Supplemental Figure $1, \mathrm{~A}^{-} \mathrm{C}$ ).

To analyze CUL4B function in islet circuits, we crossed $\mathrm{Cul} 4 \mathrm{~b}^{\mathrm{fl} / \mathrm{fl}}$ mice with Ins2-Cre transgenic mice or Sst-Cre transgenic mice to generate cell type-specific Cul4b-knockout mice (Supplemental Figure $1, \mathrm{D}-\mathrm{F})$. Both cell type-specific knockout mice were born at expected Mendelian frequencies and exhibited normal life expectancies. Compared with those in Ins2-Cre mice, CUL4B protein expression levels were decreased by $80 \%$ in the islets of Ins2-Cre ${ }^{+/-} \mathrm{Cul} 4 \mathrm{~b}^{f / Y}$ mice, as confirmed by Western blotting and immunofluorescence analysis (Supplemental Figure 1, G and H). Insulin and somatostatin immunofluorescence showed that Ins2- $\mathrm{Cre}^{+/-} \mathrm{Cul} 4 b^{f / Y}$ mice had the same number of islets and comparable $\beta$ and $\delta$ cell numbers (Supplemental Figure 2, A and B). Furthermore, no significant differences in fasting and fed glucose levels or glucose tolerance were observed between the Ins2-Cre ${ }^{+/-} \mathrm{Cul} 4 \mathrm{~b}^{f / Y}$ mice and the Ins2-Cre ${ }^{+/-}$mice (Supplemental Figure 2, C and D).

We next investigated the specific functions of CUL4B in somatostatin-secreting cells using Sst-Cre ${ }^{+/-} \mathrm{Cul}_{4} b^{t / Y}$ mice (Supplemental Figure 1, D and E). CUL4B was not detected in the pancreatic

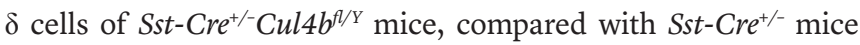
(referred to as WT), by immunostaining (Figure 1D and Supple- mental Figure 1E). Immunofluorescence also showed no significant differences in islet or $\beta$ or $\delta$ cell numbers between $\mathrm{Sst}-\mathrm{Cre}^{+/-}$ $\mathrm{Cul}_{4} \mathrm{~b}^{t / Y}$ and Sst-Cre $\mathrm{C}^{+/-}$mice (Figure 1, E and F). We then examined glucose homeostasis in Sst-Cre ${ }^{+/-} \mathrm{Cul} 4 \mathrm{~b}^{f / /}$ mice. Although Sst-Cre $\mathrm{Cr}^{+/-}$ $C u l 4 b^{t / Y}$ mice exhibited fasting glucose levels comparable to those of Sst-Cre ${ }^{+/-}$mice, the knockout mice exhibited increased glucose levels after 2 hours of feeding following a 16-hour fast (Figure 1G). Accordingly, during a glucose tolerance test, the blood glucose levels of Sst-Cre ${ }^{+/-} \mathrm{Cul}_{4} \mathrm{~b}^{t / Y}$ mice were $33 \%$ and $30 \%$ higher at $30 \mathrm{~min}-$ utes and 120 minutes, respectively, than those of the control group consisting of both $\mathrm{Sst}-\mathrm{Cre}^{+/-}$and $\mathrm{Cul} 4 \mathrm{~b}^{f / Y}$ mice (Figure $1 \mathrm{H}$ ). In addition, during the insulin tolerance test, the blood glucose levels of Sst-Cre ${ }^{+/-} \mathrm{Cul} 4 \mathrm{~b}^{f / Y}$ mice unexpectedly decreased more dramatically and rapidly than those of the $\mathrm{Sst}-\mathrm{Cre}^{+/-}$control mice in response to insulin stimulation (Figure 1I). Insulin and somatostatin content as well as kidney, brain, heart, liver, and stomach weights were not found to be significantly different between the $\mathrm{Sst}-\mathrm{Cre}^{+/-} \mathrm{Cul} 4 \mathrm{~b}^{f / Y}$ mice and their Sst-Cre ${ }^{+/-}$littermates (Supplemental Figure 2, E-G). Together, these data indicate that ablation of CUL4B in mouse $\delta$ cells, but not $\beta$ cells, impairs glucose tolerance.

Ablation of CUL $4 B$ in $\delta$ cells impairs glucose-mediated insulin secretion by increasing somatostatin secretion. The impaired glucose metabolism found in the $\mathrm{Sst}$ - $\mathrm{Cre}^{+/-} \mathrm{Cul} 4 \mathrm{~b}^{f / \mathrm{Y}}$ mice was probably due to dysfunctional hormone secretion from the pancreatic islets. Therefore, we detected levels of plasma and secreted insulin and somatostatin in isolated islets from Sst-Cre ${ }^{+/-} \mathrm{Cul} 4 \mathrm{~b}^{t / Y}$ and Sst-Cre ${ }^{+/-}$mice. After an overnight fast, the plasma insulin levels of Sst-Cre ${ }^{+/-} \mathrm{Cul} 4 \mathrm{~b}^{f / Y}$ mice showed no significant differences compared with those of Sst-Cre $\mathrm{C}^{+/}$mice (Figure 2A). After a 2-hour feeding period, plasma insulin levels increased 3.7-fold in the Sst$\mathrm{Cre}^{+/-}$mice, whereas plasma insulin levels of $\mathrm{Sst}-\mathrm{Cre}^{+/-} \mathrm{Cul} 4 \mathrm{~b}^{\mathrm{fl} / \mathrm{Y}}$ mice increased by approximately 2-fold (Figure 2A). However, we found no significant differences in plasma somatostatin levels of fasted versus fed animals in both $\mathrm{Sst}-\mathrm{Cr} \mathrm{e}^{+/-} \mathrm{Cul} 4 \mathrm{~b}^{\mathrm{t} / \mathrm{Y}}$ and $\mathrm{Sst}-\mathrm{Cr} \mathrm{C}^{+/-}$ mice (Figure 2B). We then isolated pancreatic islets from Sst- $\mathrm{Cre}^{+/-}$ $\mathrm{Cul}_{4} \mathrm{~b}^{t / \mathrm{Y}}$ and $\mathrm{Sst}$-Cre $\mathrm{Cr}^{+/-}$mice and examined insulin and somatostatin secretion patterns in response to $1 \mathrm{mM}, 5.5 \mathrm{mM}$, and $20 \mathrm{mM}$ glucose or high $\mathrm{KCl}$ stimulation conditions. High glucose levels induced both insulin and somatostatin secretion from isolated islets (Figure 2, C-F). However, glucose-induced insulin secretion was $47 \%$ lower in islets isolated from $\mathrm{Sst}-\mathrm{Cre}^{+/-} \mathrm{Cul} 4 \mathrm{~b}^{t / Y}$ mice than in those from the control Sst-Cre ${ }^{+/-}$mice (Figure 2, C and E). In contrast, glucose-induced somatostatin levels were found to be $140 \%$ higher than those in control mice (Figure 2, D and F). High potassium levels also induced insulin and somatostatin secretion from isolated islets, and islets from Sst-Cre ${ }^{+/-} \mathrm{Cul}_{4} \mathrm{~b}^{f / \mathrm{Y}}$ mice exhibited significantly decreased insulin secretion and corresponding increases in somatostatin (Figure 2, E and F). We then examined glucose-induced insulin and somatostatin secretion trends over different stimulation time points. The islets from Sst$\mathrm{Cre}^{+/-} \mathrm{Cul} 4 \mathrm{~b}^{f l / Y}$ mice compared with the islets from Sst-Cre mice exhibited decreased insulin secretion levels after both short- and long-term glucose exposure (Figure $2 \mathrm{G}$ ). In contrast, glucoseinduced somatostatin secretion from islets of Sst-Cre ${ }^{+/-} \mathrm{Cul} 4 \mathrm{~b}^{f / Y}$ mice was $140 \%$ and $40 \%$ higher than that of Sst-Cre ${ }^{+/-}$mice at 5 minutes and 60 minutes, respectively (Figure $2 \mathrm{H}$ ). In contrast to the results found for the Sst-Cre ${ }^{+/-} \mathrm{Cul} 4 b^{t / Y}$ mice, CUL4B defi- 
A
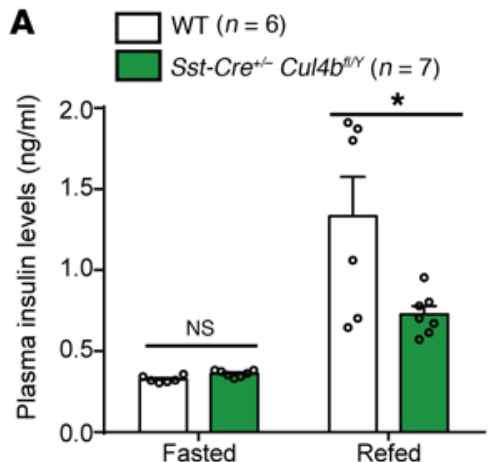

D

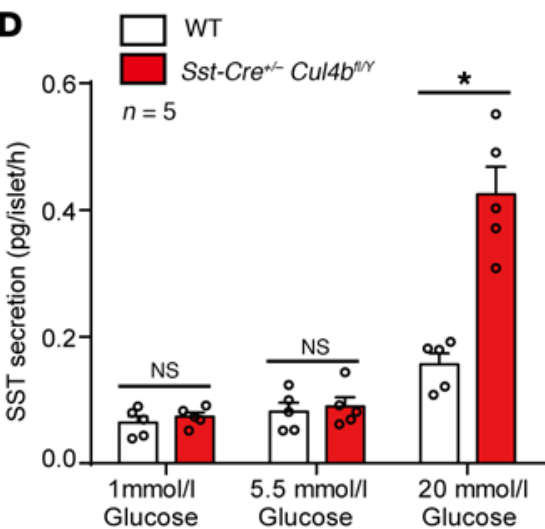

B

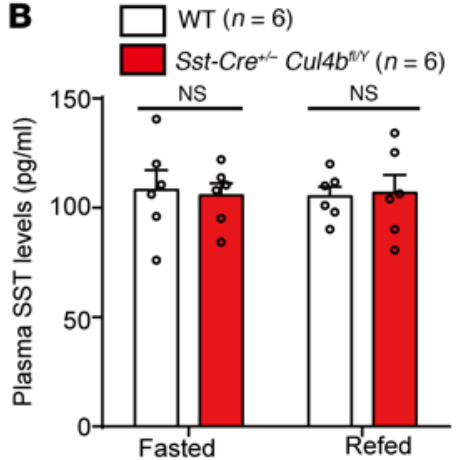

E

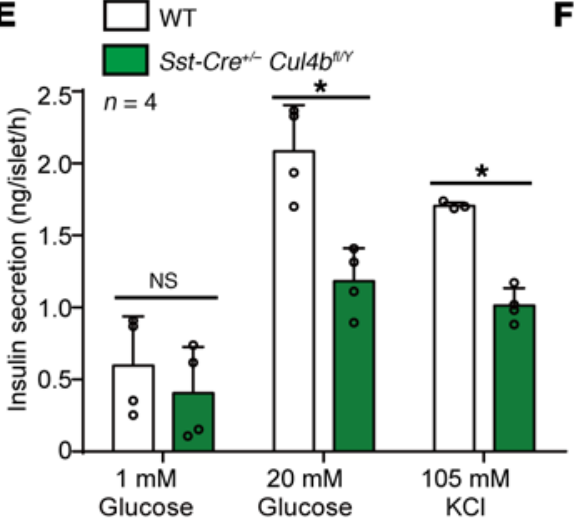

$\mathbf{F}$
C $\square$ wT
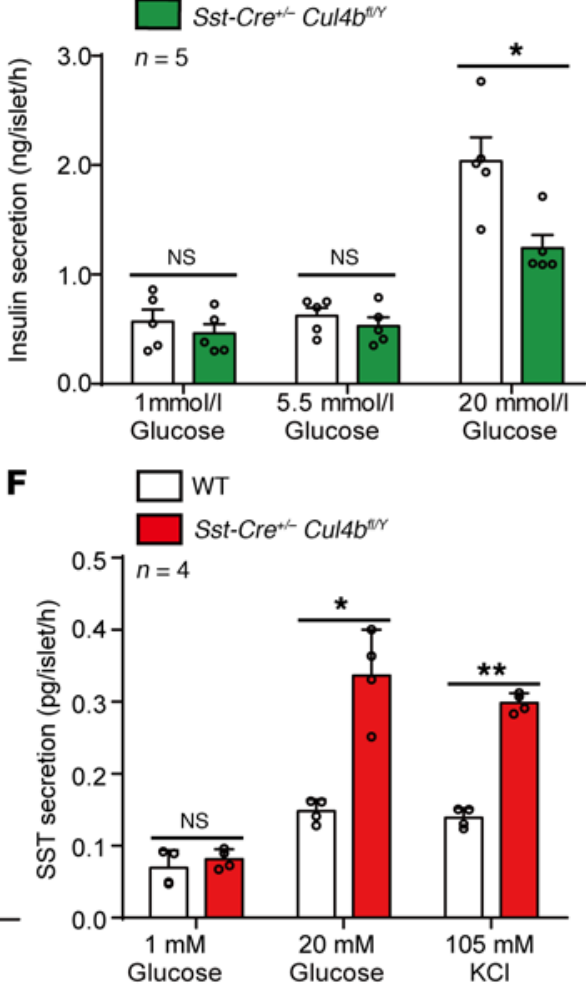

G $\square$ wT

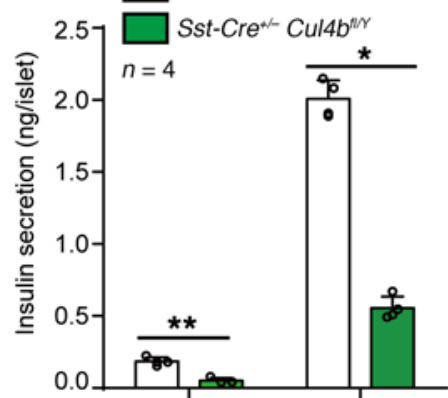

H
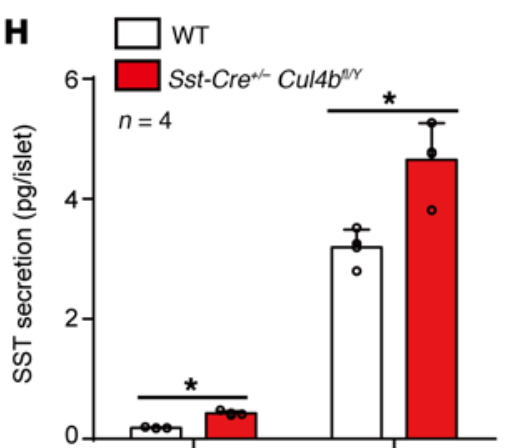

$20 \mathrm{mM}$ Glucose $5 \mathrm{~min}$

Time (min)

60 min

$20 \mathrm{mM}$ Glucose $5 \mathrm{~min}$

Time (min)
$60 \mathrm{~min}$

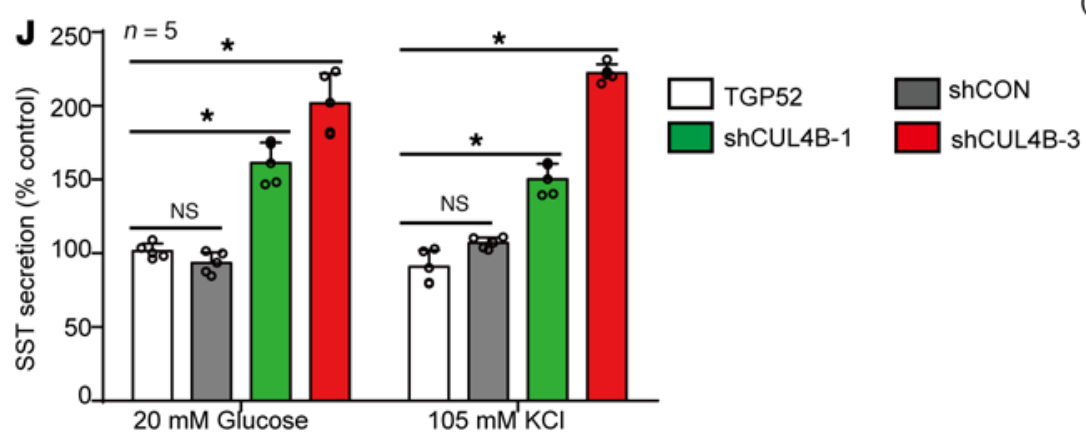

I

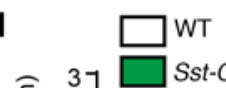

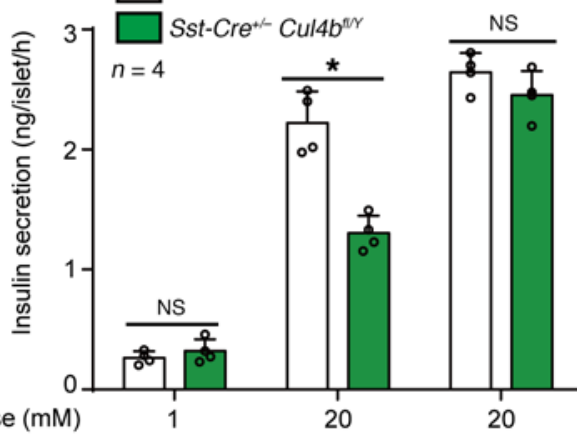

Glucose (mM)

Cyclosomatostatin $(12 \mu \mathrm{M})$

Figure 2. Impaired glucose tolerance in Sst-Cre ${ }^{+-} \mathbf{C u l 4 b ^ { f / Y }}$ mice is due to increased somatostatin paracrine signaling. (A and B) Plasma insulin levels (A) and somatostatin levels (B) of WT and Sst-Cre ${ }^{+/-}$Cul4 $b^{f l / \gamma}$ mice in the fasted and fed states. $n=6-7$ mice per group. (C and D) 1 mM, 5.5 mM, and 20 mM glucoseinduced insulin (C) and somatostatin (D) secretion in islets isolated from Sst-Cre ${ }^{+/-}$Cul $4 b^{f l / \gamma}$ mice and WT littermates after 5 minutes. $n=5$ mice per group. (E and F) Glucose- and high $\mathrm{KCl}$-induced insulin secretion (E) or somatostatin secretion (F) of islets isolated from WT and Sst-Cre ${ }^{+/-}$Cul4 $b^{f / / \gamma}$ mice after 5 minutes. $n=4$ mice per group. (G and $\mathbf{H}$ ) Short- and long-term glucose-induced insulin secretion (G) and somatostatin secretion (H) of islets isolated from WT mice and Sst-Cre ${ }^{+/-}$Cul $4 b^{f / Y}$ mice. $n=4$ mice per group. (I) Effect of the somatostatin receptor antagonist cyclosomatostatin (12 $\mu \mathrm{M}$ ) on glucose-induced insulin secretion of islets isolated from Sst-Cre ${ }^{+/-}$Cul4 $b^{f / / \gamma}$ mice and WT littermates after 5 minutes. $n=4$ mice per group. (J) Glucose- or high KCl-induced somatostatin secretion from CUL4B knockdown and control TCP52 cells after 1 hour $(n=5)$. A-I, ${ }^{*} P<0.05 ;{ }^{*} P<0.01$. Sst-Cre ${ }^{+/-}$Cul4b $b^{f / / \gamma}$ mice were compared with their WT littermates. J, ${ }^{*} P<0.05$. CUL4B knockdown cells were compared with control shRNA knockdown cells. Bars represent mean \pm SEM. All data were analyzed using 1-way ANOVA. 
ciency in the pancreatic $\beta$ cells of Ins2-Cre ${ }^{+/-} \mathrm{Cul} 4 \mathrm{~b}^{f / \mathrm{Y}}$ mice did not significantly affect insulin and somatostatin secretion levels in response to high glucose relative to trends found for Ins2-Cre ${ }^{+/-}$ mice (Supplemental Figure 3, A and B).

It was reported that somatostatin inhibits glucose-induced insulin secretion from islet $\beta$ cells by activating $G$ protein-coupled somatostatin receptors $(32,35)$. In agreement with previous results, our data showed that somatostatin behaved as a paracrine regulator of glucose metabolism and insulin secretion in Sst-Cre $\mathrm{Cr}^{+/-}$ $C u l 4 b^{f / Y}$ mice. Downregulation of insulin secretion from pancreatic islets of $\mathrm{Sst}-\mathrm{Cre}^{+/-} \mathrm{Cul} 4 \mathrm{~b}^{f / Y}$ mice may have resulted from the increased levels of somatostatin release. To this end, we pretreated islets with cyclosomatostatin, a general somatostatin receptor antagonist, and we then stimulated them under high glucose levels. The administration of cyclosomatostatin abolished differential glucose-induced insulin secretion observed between $\mathrm{Sst}-\mathrm{Cr}^{+/-}$ $\mathrm{Cul}_{4} \mathrm{~b}^{f l / Y}$ and Sst-Cre $\mathrm{C}^{+/-}$pancreatic islets (Figure 2I). To further confirm the direct regulatory role of CUL4B in pancreatic $\delta$ cell functioning, we used the in vitro TGP52 cell system and knocked down CUL4B by shRNA. TGP52 is a pancreatic epithelial cell line that secretes somatostatin only rather than insulin in response to high glucose or high potassium stimulation (Supplemental Figure 4, A and B) (36). Two TGP52 stable cell lines treated with different shRNAs showed significantly decreased expression levels of CUL4B (Supplemental Figure 4, C and D). In agreement with our in vivo results, TGP52 cells with lower CUL4B expression levels secreted more somatostatin after high glucose or high potassium stimulation (Figure 2J).

Elevated calcium and cAMP levels in $\delta$ cells contribute to enhanced somatostatin secretion. ATP-sensitive potassium (KATP) channels are key players in the regulation of insulin and somatostatin secretion from pancreatic $\beta$ and $\delta$ cells, respectively, because activation of KATP channels completely abolishes glucose-induced insulin and somatostatin secretion $(5,37,38)$. To investigate the mechanisms underlying increased somatostatin secretion levels found in Sst-Cre ${ }^{+/-} \mathrm{Cul}_{4} b^{f / Y}$ mice, we first examined the effects of diazoxide, a specific KATP channel opener, on glucose-induced somatostatin secretion in islets isolated from $\mathrm{Sst}-\mathrm{Cre}^{+/-} \mathrm{Cul} 4 \mathrm{~b}^{f / Y}$ mice or $\mathrm{Sst}-\mathrm{Cre}^{+/-}$littermates. Treatment with diazoxide inhibited somatostatin secretion in islets isolated from both $\mathrm{Sst}-\mathrm{Cre}^{+/-} \mathrm{Cul} 4 \mathrm{~b}^{t / Y}$ and control mice to a similar extent, thus indicating that increased somatostatin secretion was not due to changes in KATP channel activity in pancreatic $\delta$ cells (Figure 3A). In contrast, diazoxide inhibited glucose-induced insulin secretion to the same extent in islets isolated from $\mathrm{Sst}-\mathrm{Cre}^{+/-} \mathrm{Cul} 4 b^{f l / Y}$ mice and from their WT littermates, possibly because of the common essential downstream pathway mediated by KATP channels governing insulin secretion in pancreatic $\beta$ cells in mice of both genotypes (Figure 3B).

Recent studies have shown that L-type and R-type calcium channels together with the ryanodine receptor pathway are involved in glucose-induced somatostatin secretion $(5,12)$. In particular, the application of the L-type channel blocker isradipine abolished the potentiation effects of UCN3 on glucose-stimulated somatostatin secretion from pancreatic $\delta$ cells (5). Therefore, we examined glucose-induced islet somatostatin secretion in the absence or presence of the R-type channel blocker SNX482, the L-type channel blockers nicardipine and isradipine, and the endoplasmic reticulum $\mathrm{Ca}^{2+}$ ATPase inhibitor thapsigargin (Figure 3C). Similarly to what was recently reported (12), glucoseinduced somatostatin secretion was significantly blocked by R-type channel antagonist SNX482 (Figure 3C). However, even

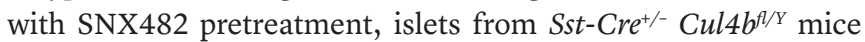
still released somatostatin levels twice as high as those of their WT littermates, indicating that the increased somatostatin levels found in the knockout islets did not form because of changes in the opening of R-type calcium channels. Interestingly, when we blocked L-type calcium channels with nicardipine or isradipine, we found that islets isolated from Sst-Cre ${ }^{+/-} \mathrm{Cul} 4 b^{f / / Y}$ mice released the same amount of somatostatin as those from control mice (Figure $3 \mathrm{C}$ ). These results showed that L-type calcium channels play an important role in increased somatostatin secretion in Sst-Cre $\mathrm{Cr}^{+/-}$ $C u l 4 b^{f / Y}$ mice. We then used TGP52 pancreatic $\delta$ cells to identify a direct interaction between CUL4B and L-type calcium channels in somatostatin secretion of pancreatic $\delta$ cells. Indeed, isradipine blocked enhanced somatostatin secretion in CUL4B knockdown TGP52 cells, whereas treatment with SNX482, thapsigagin, or diazoxide elicited insignificant effects (Supplemental Figure 5A). We next measured insulin secretion after treatment with the same panel of $\mathrm{Ca}^{2+}$ channel and ATPase inhibitors. Treatment with both the R-type calcium channel blocker SNX482 and the L-type channel inhibitor nicardipine markedly inhibited glucose-induced insulin secretion from islets. However, although the insulin secretion of islets isolated from Sst-Cre ${ }^{+/-} \mathrm{Cul} 4 \mathrm{~b}^{f / Y}$ mice was still much lower than that of their WT littermates after SNX482 treatment, this difference was eliminated after nicardipine treatment (Figure 3D). These data are in agreement with the observed somatostatin secretion patterns and reinforce the conclusion that L-type calcium channels in pancreatic $\delta$ cells are key components mediating the increased somatostatin secretion and impaired glucose metabolism in Sst-Cre ${ }^{+/-} \mathrm{Cul} 4 b^{f / Y}$ mice.

L-type calcium channels are responsible for the influx of extracellular calcium during hormone secretion. We therefore measured high glucose- and high potassium-induced calcium signals in isolated primary pancreatic $\delta$ cells from $S s t-\mathrm{Cre}^{+/-} \mathrm{GFP}^{f /+} \mathrm{Cul}^{\mathrm{f}} \mathrm{b}^{f / Y}$ mice and their WT littermates. The pancreatic $\delta$ cells derived from Sst$\mathrm{Cre}^{+/-} \mathrm{GFP}^{f l /+} \mathrm{Cul} 4 \mathrm{~b}^{f / Y}$ mice showed significantly higher frequencies of high glucose-induced calcium signals than the WT primary pancreatic $\delta$ cells (Figure 3, E and F). Similarly, in pancreatic $\delta$ cells derived from $\mathrm{Sst}-\mathrm{Cre}^{+/-} \mathrm{GFP}^{f /+} \mathrm{Cul}_{4} \mathrm{~b}^{f / \mathrm{Y}}$ mice, potassium-induced calcium increases were significantly greater than those in the WT littermates (Figure 3, G and H). However, after isradipine pretreatment, intracellular calcium signals of the Sst-Cre ${ }^{+/-} \mathrm{GFP}^{f / /+} \mathrm{Cul}^{\mathrm{f}} \mathrm{b}^{f / \mathrm{Y}}$ mice were decreased to $50 \%$, reflecting patterns found for the WT (Figure $3, \mathrm{G}$ and $\mathrm{H})$. Therefore, intracellular calcium increases contribute to increased somatostatin secretion in Sst-Cre ${ }^{+/-} \mathrm{GFP}^{f /+} \mathrm{Cul}^{f / b^{f / Y}}$ mice and are dependent on L-type calcium channel activity.

In addition to intracellular calcium, the second messenger cAMP, the $\mathrm{G}_{\mathrm{q}}$-PLC pathway, and kinase signaling are actively involved in hormone secretion from pancreatic islets $(5,13,39-$ 41). Therefore, we preincubated the islets with several inhibitors, including cAMP-PKA signaling inhibitors Rp-CAMPs and H89, $G_{\mathrm{q}}$-PLC inhibitor U73122, MEK inhibitor U0126, and adenyl cyclase (AC) inhibitor 2',5'-dideoxyadenosine (DDA), and we examined glucose-induced somatostatin secretion patterns 

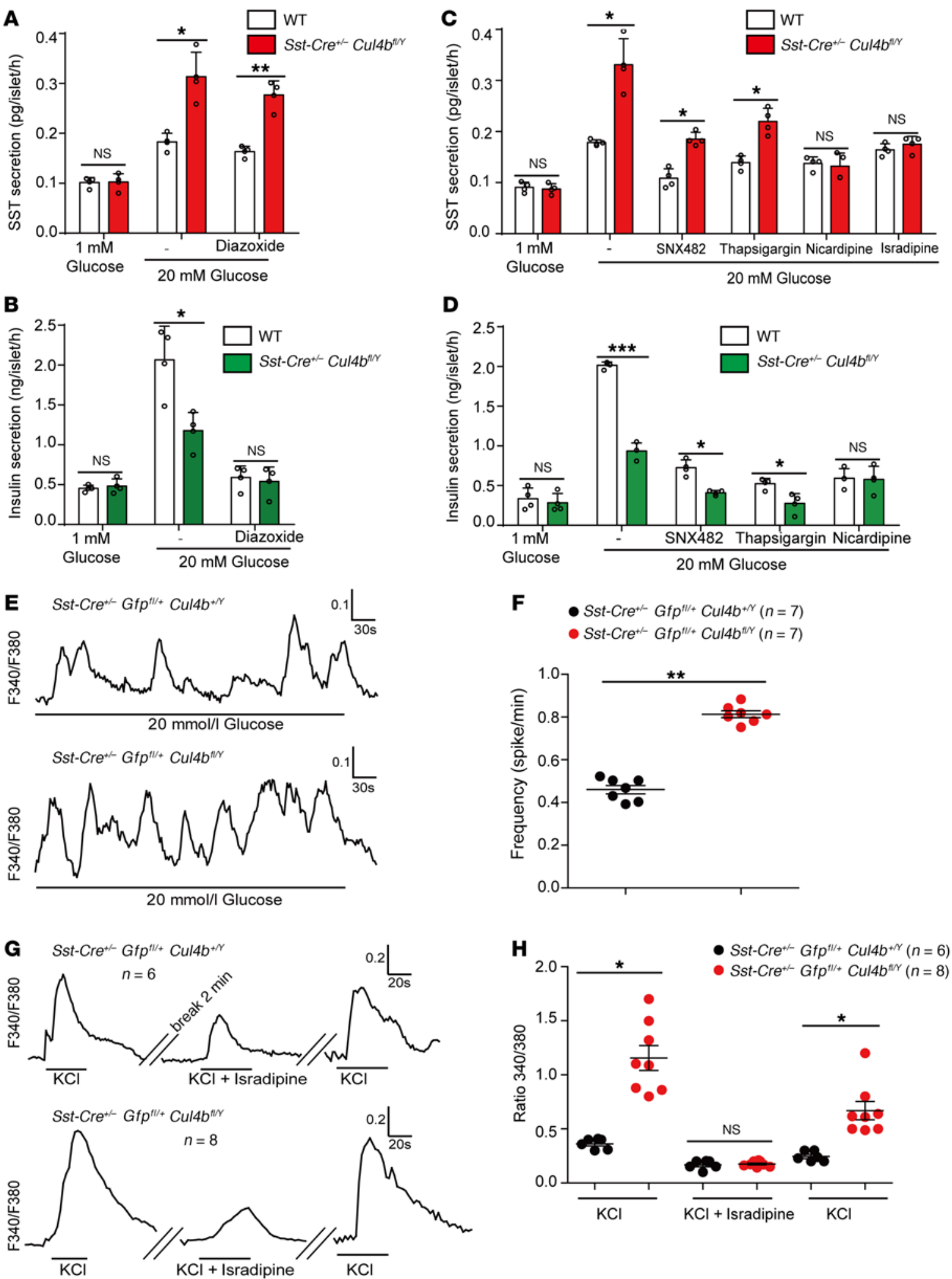

Figure 3. L-type calcium channels play key roles in enhanced somatostatin secretion from CUL4B-deficient $\delta$ cells. (A) Effect of diazoxide (250 $\mu$ M), a KATP channel opener, on glucose-induced somatostatin secretion in islets isolated from Sst-Cre ${ }^{+/-}$Cul $4 b^{f / \gamma}$ mice and WT littermates after 5 minutes. ( $n=4$ ). (B) Effect of diazoxide on glucose-induced insulin secretion in islets isolated from Sst-Cre ${ }^{+/-}$Cul $4 b^{f / / \gamma}$ or WT littermates after 5 minutes $(n=4)$. (C) Clucose-induced somatostatin secretion from islets in the absence or presence of the R-type channel blocker SNX482 (100 nM) and the L-type channel blockers isradipine (10 $\mu \mathrm{M})$ and nicardipine $(10 \mu \mathrm{M})$ after 5 minutes $(n=4)$. (D) Glucose-induced insulin secretion from islets in the absence or presence of an R-type channel blocker or L-type channel blockers after 5 minutes $(n=4)$. ( $(\mathbf{D}$ and $\mathbf{F})$ Representative curves and statistical analysis of a high glucose-induced calcium signal in isolated pancreatic $\delta$ cells from Sst-Cre ${ }^{+/-} G F P^{f / /+} C u l 4 b^{f / Y}$ mice (E, lower panel) and WT littermates (E, upper panel) $(n=8)$. (G and $\left.\mathbf{H}\right)$ Representative curves and statistical analysis of high $\mathrm{KCl}$-induced calcium signal in $\delta$ cells from Sst-Cre ${ }^{+/-} \mathrm{GFPf/+} C u l 4 b^{f / \gamma}$ mice (G, lower panel) or WT littermates (G, upper panel) $(n=8)$. ${ }^{*} P<0.05$; ${ }^{* *} P<0.01$; ${ }^{* * *} P<0.001$. Sst-Cre ${ }^{+/-} G F P^{f /+}$ Cul4b $b^{f / /}$ mice were compared with their WT littermates. Bars represent mean \pm SEM. All data were analyzed using 1-way ANOVA. 
A

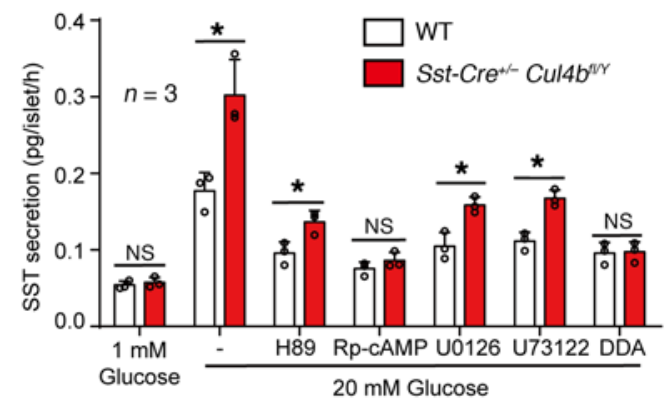

C

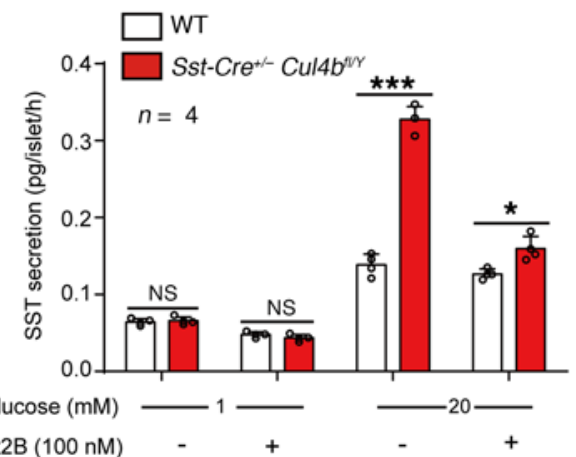

B
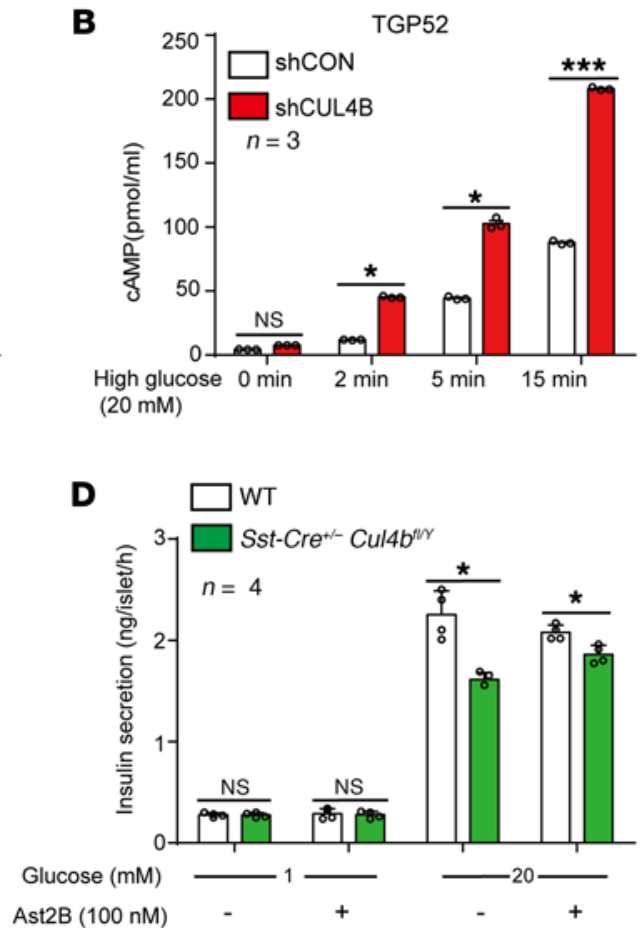

Figure 4. CRHR2 activity and the CAMP signaling pathway are important in increased somatostatin secretion. (A) Clucose-induced somatostatin secretion in islets isolated from WT and Sst-Cre ${ }^{+/-}$Cul4 $b^{f / \gamma}$ mice treated with inhibitors for PKA (H89: $\left.10 \mu \mathrm{M}\right)$, cAMP (Rp-CAMP: $\left.100 \mu M\right)$, ERK (U0126: $10 \mu M$ ), PLC (U73122: $10 \mu \mathrm{M})$, or AC (DDA: $100 \mu \mathrm{M})$ after 5 minutes $(n=3)$. (B) Glucose-induced cAMP signals in CUL4B knockdown and control TCP52 cells $(n=4)$. (C-D) Effects of Ast2B on glucose-induced insulin (D) and somatostatin (C) secretion in islets isolated from Sst-Cre ${ }^{+/-}$Cul4 $4 b^{f / / \gamma}$ or WT littermates after 5 minutes $(n=4)$. A, C, D, ${ }^{*} P<0.05 ;{ }^{* * *} P<0.001$. Sst-Cre ${ }^{+/-}$Cul4 $b^{f / /}$ mice were compared with their WT littermates. B, ${ }^{*} P<0.05 ;{ }^{* * *} P<0.001$. CUL4B knockdown cells were compared with control shRNA knockdown cells. Bars represent mean \pm SEM. All data were analyzed using 1-way ANOVA.

(Figure 4A). Interestingly, cAMP antagonist Rp-cAMPs and AC inhibitor DDA completely blocked enhanced somatostatin secretion from the islets of $\mathrm{Sst}-\mathrm{Cre}^{+/} \mathrm{Cul} 4 \mathrm{~b}^{\mathrm{f} / \mathrm{Y}}$ mice compared with their WT littermates whereas no significant effects were found for the other inhibitors. Moreover, treatment with cAMP antagonist RpcAMPs completely eliminated enhanced somatostatin secretion in Cul4b knockdown TGP52 cells compared with control cells (Supplemental Figure 5B). These results suggest that the cAMP pathway actively participates in the increased somatostatin secretion observed in Sst-Cre ${ }^{+-} \mathrm{Cul}_{4} b^{f / Y}$ mice. We therefore measured intracellular cAMP levels in CUL4B knockdown TGP52 and control cells. High glucose levels elicited time-dependent intracellular cAMP accumulation; intracellular cAMP levels in the CUL4B knockdown TGP52 cells increased 4-fold, 3-fold, and 3-fold over 2 minutes, 5 minutes, and 15 minutes, respectively (Figure 4B).

Pancreatic islet $\delta$ cells receive the UCN3, a paracrine signal from pancreatic $\beta$ cells, which consequently regulates somatostatin secretion, potentially through Gs-cAMP signaling (5). We therefore explored whether UCN3 and its receptor CRHR2 participate in the hypersecretion of somatostatin in $\mathrm{Sst}-\mathrm{Cre}^{+/-} \mathrm{Cul} 4 b^{\mathrm{A} / \mathrm{Y}}$ mice. Interestingly, CRHR2 antagonist astressin 2B (Ast2B) significantly decreased glucose-stimulated somatostatin secretion from islets isolated from Sst-Cre ${ }^{+/} \mathrm{Cul} 4 b^{f / Y}$ mice, whereas it was not found to have a significant effect on islets isolated from their WT littermates (Figure 4C). Correspondingly, high levels of glucose-induced insulin secretion from Sst-Cre ${ }^{+/-} \mathrm{Cul} 4 b^{f / Y}$ mice were restored to levels similar to those of the control WT mice via the application of Ast2B (Figure 4D).
Together, these results show that increased intracellular calcium and cAMP levels in pancreatic $\delta$ cells contribute to enhanced somatostatin secretion and impaired glucose homeostasis in Sst$\mathrm{Cre}^{+/-} \mathrm{Cul}_{4} \mathrm{~b}^{\mathrm{f} / \mathrm{Y}}$ mice. Specifically, the UCN3 receptor CRHR2 and L-type calcium channels play important roles in the increased levels of somatostatin secretion in $\mathrm{Sst}-\mathrm{Cr}{ }^{+/-} \mathrm{Cul} 4 \mathrm{~b}^{f / \gamma}$ mice.

Transcriptional upregulation of Cav1.2 and AC6 contributes to enhanced somatostatin secretion in CUL $4 B$-deficient pancreatic $\delta$ cells. To investigate the molecular mechanisms underlying the increased intracellular calcium and cAMP levels in response to high glucose and high potassium levels in CUL4B-deficient mice, we measured the mRNA levels of calcium channels, adenylyl cyclases, $G$ proteins, PKA, and transcription factors in CUL4B knockdown and control TGP52 cells (Figure 5A). Specifically, mRNA levels of Cav1.2, Cav1.3, AC6, and AC7 increased in CUL4B knockdown cells, and the mRNA level of hematopoietically expressed homeobox (Hhex) decreased. Consistent with upregulation of transcription, the protein levels of Cav1.2, Cav1.3, and AC6 in the CUL4B knockdown cells were higher than those in the control cells (Figure 5, B-E). To confirm that the expression of these specific calcium channels and adenylyl cyclases was regulated by CUL4B in a physiological context, we measured mRNA levels of the $\alpha$ subunits of L-type calcium channels and different adenylyl cyclases in primary $\delta$ cells isolated from Sst-Cre ${ }^{+/-} \mathrm{GFP}^{\mathrm{Al}+} \mathrm{Cul}^{\mathrm{A} \mathrm{b}^{\mathrm{A} / \mathrm{Y}}}$ mice and their WT littermates (Figure 5F and Supplemental Figure 12). These results showed that the expression of key regulators of extracellular calcium 

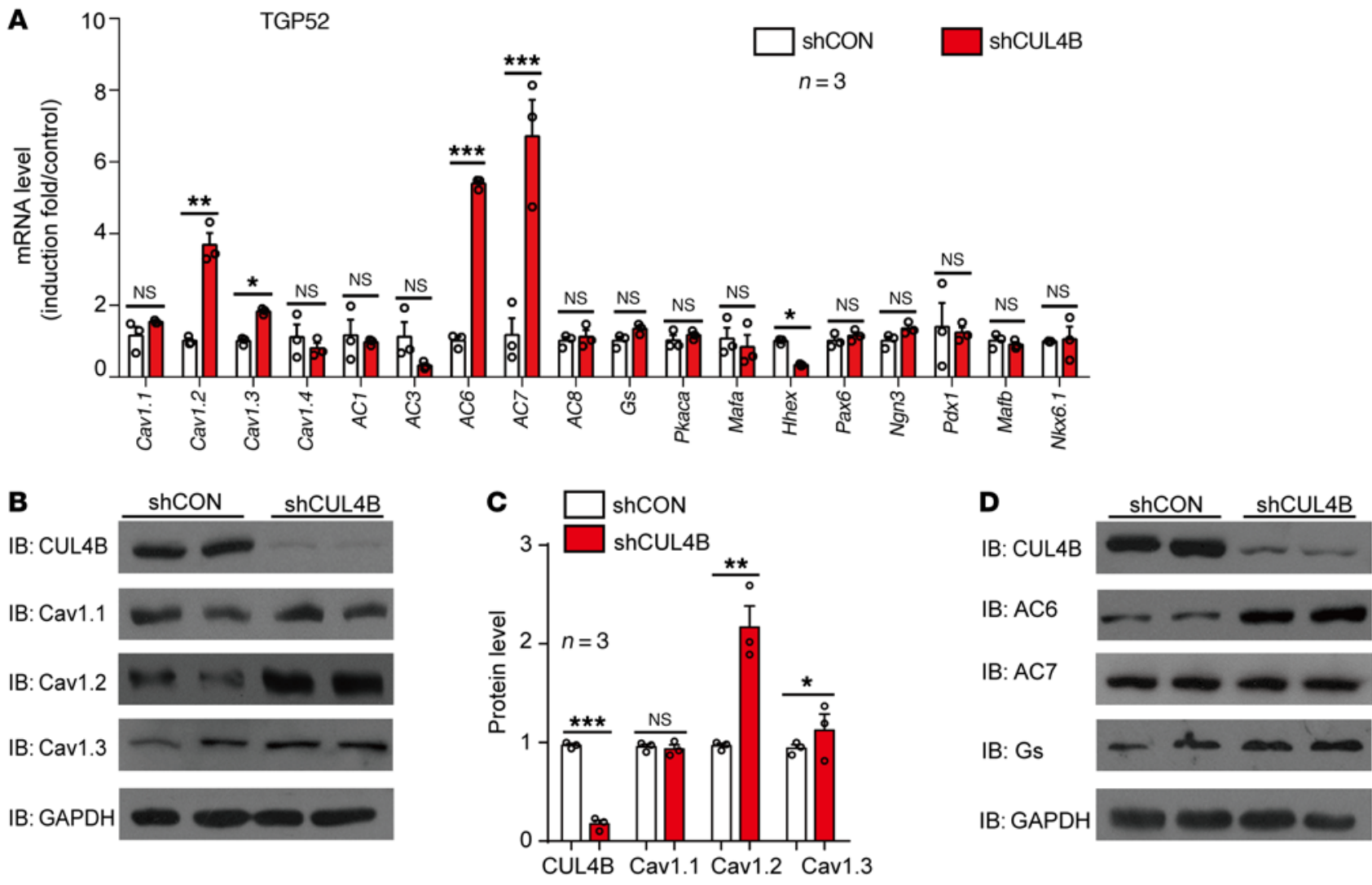

E

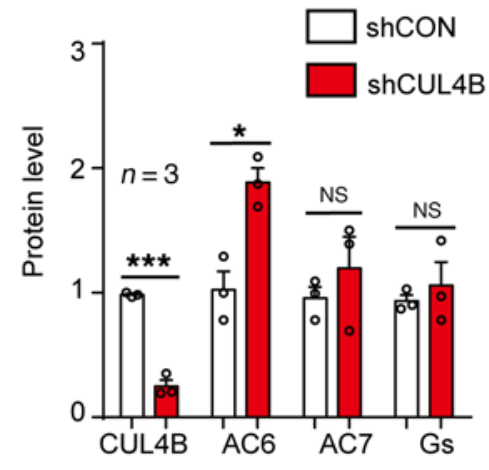

G

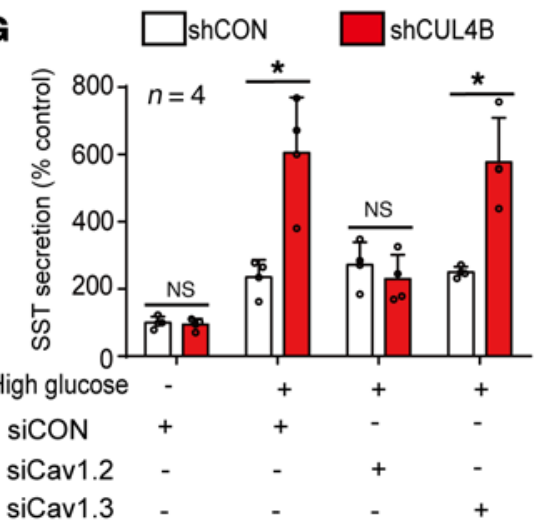

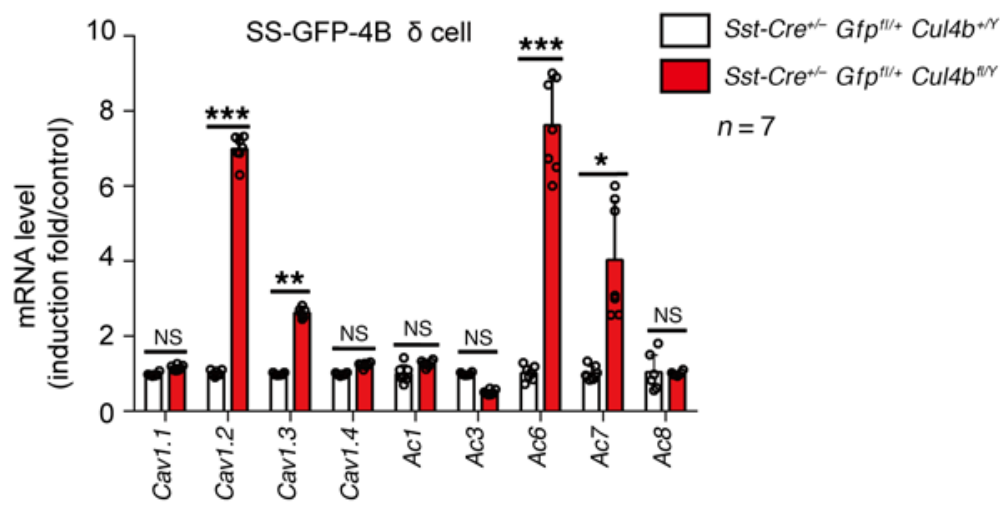

H

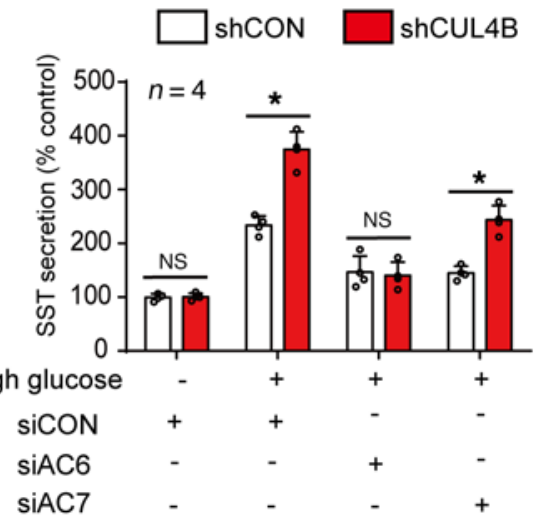

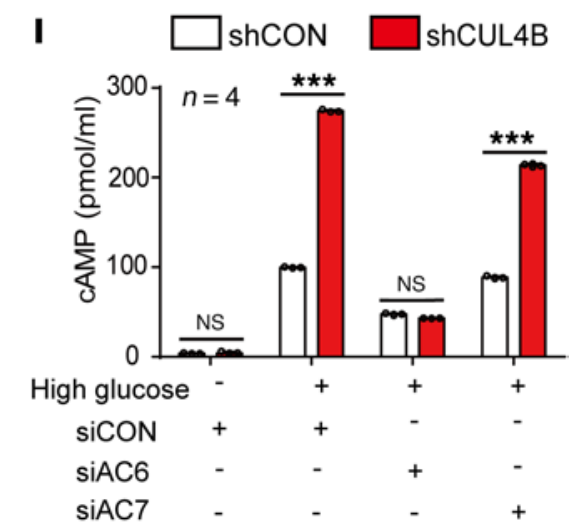


Figure 5. Transcriptional upregulation of Cav1.2 and AC6 contributes to the enhanced somatostatin secretion in Cul4b-deficient pancreatic $\delta$ cells. (A) The mRNA levels of L-type calcium channels, adenylyl cyclases, $G$ proteins, PKA and transcription factors in CUL4B knockdown and control TCP52 cells, as determined by quantitative reverse-transcriptase PCR (qRT-PCR) $(n=3)$. (B-E) Cav1.1, Cav1.2, Cav1.3, AC6, AC7 and Gs protein levels in CUL4B knockdown and control TGP52 cells. B and D, representative Western blots from at least $\mathbf{3}$ experiments are shown. $\mathbf{C}$ and $\mathbf{E}$, bar graph representation and statistical analyses of $\mathbf{B}$ and $\mathbf{D}$, respectively. $(\mathbf{F})$ qRT-PCR was used to analyze the mRNA levels of L-type calcium channel $\alpha$ subunits and adenylyl cyclases in primary $\delta$ cells from $\mathrm{Sst}-\mathrm{Cre}^{+/-} \mathrm{CFPf//+}$ Cul $4 b^{f / Y}$ and WT littermates $(n=7)$. (G) Effects of Cav1.2 or Cav1.3 knockdown on somatostatin secretion from CUL4B knockdown and control TCP52 cells $(n=4)$. (H) Effects of AC6 or AC7 knockdown on somatostatin secretion from CUL4B knockdown and control TCP52 cells. (I) Glucoseinduced cAMP levels in AC6 or AC7 knockdown and control TCP52 cells, with or without CUL4B knockdown. Cells were incubated with $20 \mathrm{mM}$ glucose for 15 minutes. ${ }^{*} P<0.05$; ${ }^{* *} P<0.01$; ${ }^{* *} P<0.001$. Sst-Cre ${ }^{+/-} \mathrm{GFP} P^{\mathrm{fl} /+}$ Cul $4 b^{f / / Y}$ mice were compared with their WT littermates, and CUL4B knockdown cells were compared with control shRNA-treated cells. The bars represent mean \pm SEM. All data were analyzed using 1-way ANOVA.

influx and intracellular cAMP levels, including those of Cav1.2, Cav1.3, AC6, and AC7, were increased in CUL4B-deficient cells.

To characterize the functional importance of the increased expression of specific L-type calcium channels, we knocked down Cav1.2 and Cav1.3 in CUL4B knockdown and control TGP52 cells. We found that Cav1.2, but not Cav1.3, knockdown completely eliminated enhanced somatostatin secretion (Figure 5G and Supplemental Figure 7, A-C). We next measured the effects of AC6 and $\mathrm{AC} 7$ knockdown on somatostatin secretion in CUL4B knockdown and control TGP52 cells. Both AC6 and AC7 knockdown decreased somatostatin secretion levels in CUL4B knockdown and control TGP52 cells (Figure 5H and Supplemental Figure 7, D-F). In particular, somatostatin secretion in CUL4B knockdown TGP52 cells with siAC6 was decreased to control levels whereas the somatostatin secretion of CUL4B knockdown TGP52 cells with siAC7 was still higher than that of control cells (Figure 5H). Correspondingly, only the knockdown of AC6 completely eliminated high glucose-induced cAMP elevation in CUL4B knockdown cells compared with the control cells (Figure 5I). These results showed that specific key second messenger regulators, including Cav1.2 and AC6, promote enhanced somatostatin secretion in Cul4bdeficient pancreatic $\delta$ cells.

Epigenetic mechanisms and HHEX regulation driving Cav1.2 and AC6 expression in CUL4B-deficient pancreatic $\delta$ cells. CUL4B is a known E3 ligase that directly regulates protein levels through ubiquitination $(30,42)$. To delineate how CUL4B regulates expression of Cav1.2 and AC6, we examined Cav1.2 and AC6 ubiquitination in CUL4B-overexpressing and control TGP52 cells (Figure 6A). In the presence or absence of protease inhibitor MG132, ubiquitination levels of Cav1.2 and AC6 were not increased in CUL4Boverexpressing TGP52 cells compared with control cells (Figure $6, \mathrm{~A}-\mathrm{C}$ ). These results demonstrate that CUL4B does not promote Cav1.2 and AC6 ubiquitination in pancreatic $\delta$ cells.

CRL4B has been shown to repress transcription in cooperation with the PRC2 complex (28). We then investigated whether CUL4B regulates the gene expression of Cav1.2 and AC6 through an epigenetic mechanism. AC6 has only 1 mRNA isoform, whereas
Cav1.2 has 3 mRNA-splicing isoforms at the initial transcriptional site (43) (Supplemental Figure 8A). Pancreatic $\delta$ cells primarily express Cav1.2b and Cav1.2c isoforms, which share the same promoter (Supplemental Figure 8B). The mRNA levels of these isoforms increased in CUL4B knockdown cells (Supplemental Figure $8, \mathrm{~B}-\mathrm{D}$ ). We therefore performed a quantitative ChIP (qChIP) profiling analysis of the binding patterns of CUL4B, DDB1, and the histone-lysine $\mathrm{N}$-methyltransferase EZH2 for a region located approximately $200 \mathrm{bp}$ upstream to $1,500 \mathrm{bp}$ downstream of the Cav1.2 and AC6 transcription start sites (Figure 6, D-I, and Supplemental Figure 8, E-J). The qChIP assay revealed that the occupancy sites of these proteins overlap and that the binding of CRL4B (CUL4B and DDB1) and PRC2 (EZH2) both peaked in the region at around $-1,500$ bp to $-1,219$ bp and -740 bp to -423 bp of the Cav1.2 and AC6 promoters, respectively (Figure 6, D-F, and Supplemental Figure 8, E-G). Notably, H2AK119ub1 and H3K27me3, 2 histone marks of transcription repression, were also enriched in the same region (Figure 6, G and $\mathrm{H}$ and Supplemental Figure $8, \mathrm{H}$ and I).

We next examined the effect of CUL4B depletion on the recruitment of the CRL4B-PRC2 complex to the Cav1.2 and AC6 promoters in pancreatic $\delta$ cells. Knockdown of CUL4B resulted in a marked reduction in the levels of CUL4B, DDB1, and EZH2 bound to the Cav1.2 and AC6 promoters (Figure 6, D-F, and Supplemental Figure 8, E-G). Consistently, the levels of H2AK119ub1 and H3K27me 3 were also markedly decreased at the Cav1.2 and AC6 promoters (Figure 6, G and H, and Supplemental Figure 8, $\mathrm{H}$ and I). In particular, H3K4me3 was enriched in the 1,060-bp to -900-bp region of the Cav1.2 promoter (Figure 6I). Increased H3K4me3 levels in the promoter regions of Cav1.2 and AC6 were also observed in CUL4B knockdown pancreatic $\delta$ cells, potentially contributing to elevated Cav1.2 and AC6 expression levels observed in these cells (Figure 6I and Supplemental Figure 8J). Together, these data show that CUL4B, a key component of CRL4B, represses the expression of Cav1.2 and AC6 by colocalizing with PRC2 at specific promoter regions of Cav1.2 and AC6, thus epigenetically regulating the ubiquitination and methylation of their associated histones.

Interestingly, we noticed that upregulation of Cav1.2 and AC6 are specific to CUL4B-deficient pancreatic $\delta$ cells, but not $\beta$ cells (Supplemental Figure 6, A-E). We therefore tested the connection between HHEX, a pancreatic $\delta$ cell-specific transcriptional factor essential for $\delta$ cell differentiation (11), and CUL4B-regulated Cav1.2 and AC6 expression. qChIP showed that knockdown of HHEX in TGP52 cells significantly decreased CUL4B recruitment to the promoters of Cav1.2 and AC6 (Figure 6J), suggesting that HHEX promotes the recruitment and/ or retention of the CUL4B complex at the promoters of targeted genes. We next performed a qChIP assay to profile the binding patterns of HHEX on a region of approximately 5 -kb upstream and downstream around the Cav1.2 and AC6 transcription start sites (Figure 6, $\mathrm{K}$ and L, and Supplemental Figure 10, $\mathrm{A}-\mathrm{C}$ ). The results indicated that HHEX directly binds to the DNA region of the 4,568-4,918 downstream of the transcriptional initiation site of Cav1.2 and the DNA region of 3,981 to 4,417 downstream of the initiation site of AC6 (Figure 6, K and L). Moreover, HHEX physically associated with the CRL4B-PRC2 complex in TGP52 
A
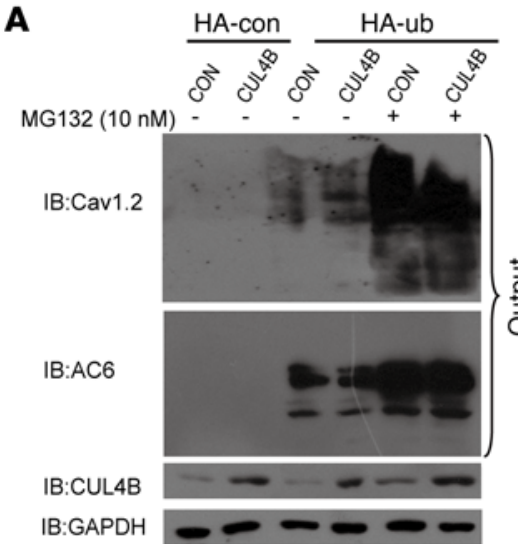

D

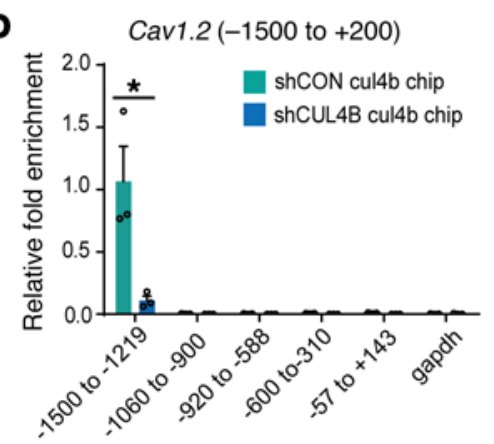

G

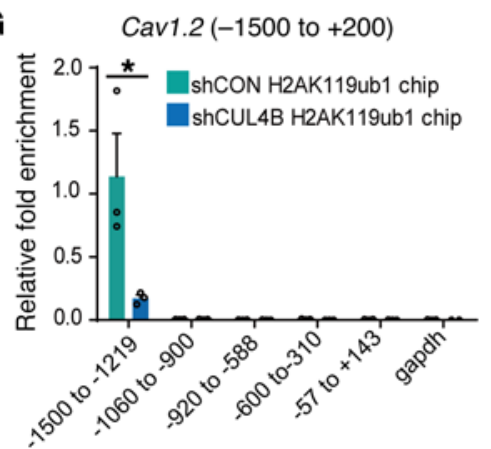

B

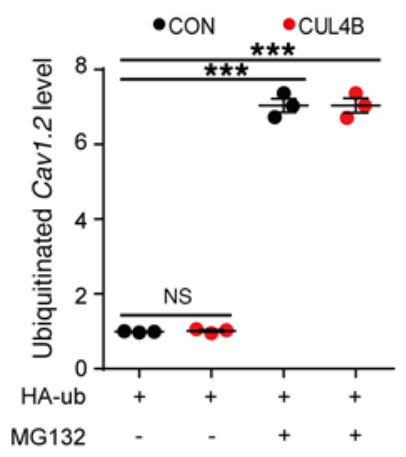

E

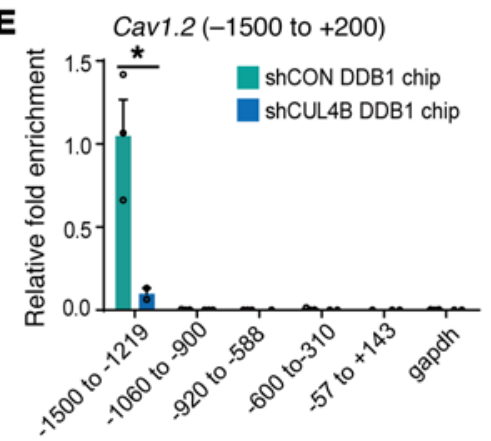

H

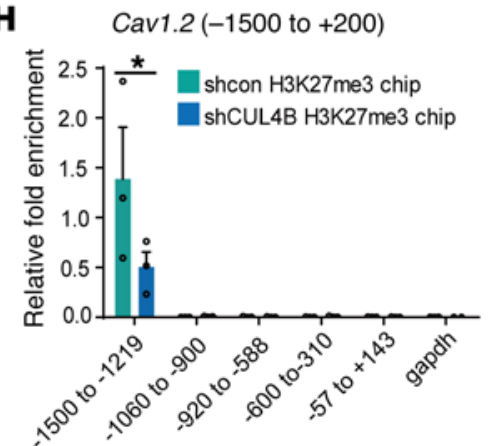

C

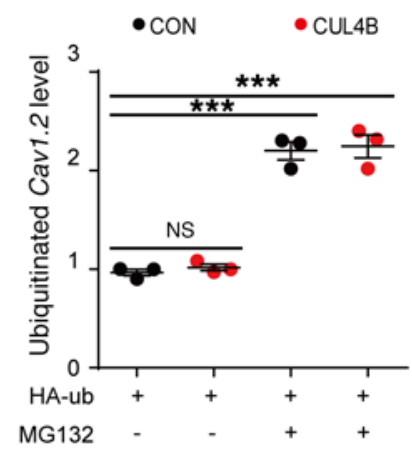

$\mathbf{F}$

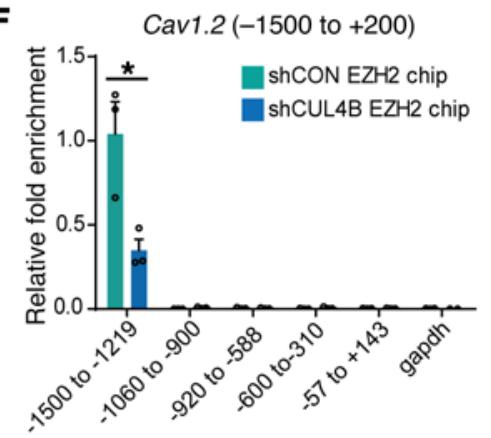

I

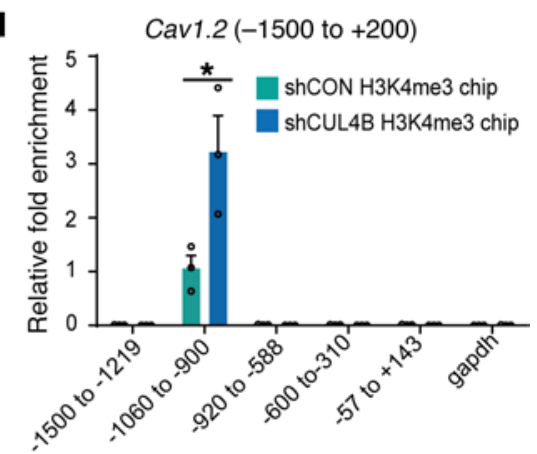

J

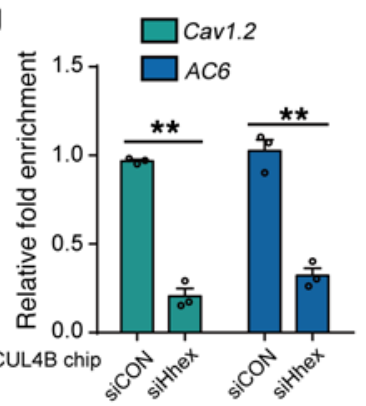

K Cav1.2(+4278 to +5278$)$

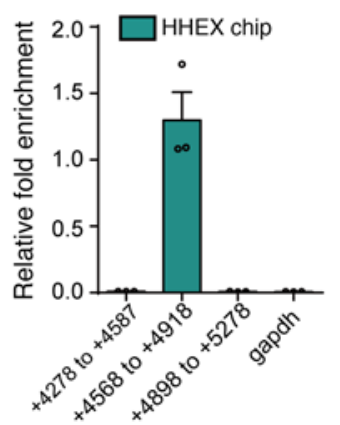

L $\quad A C 6(+3401$ to +4417$)$

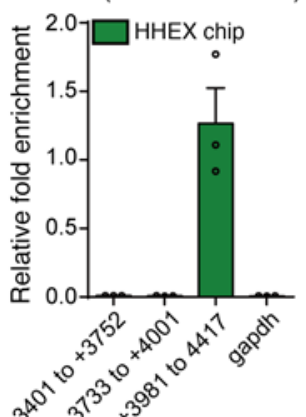

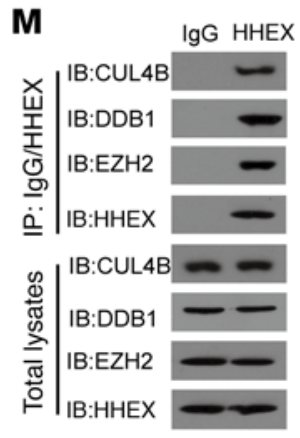


Figure 6. CUL4B negatively regulates Cav1.2 and AC6 expression in pancreatic $\delta$ cells through an epigenetic mechanism. (A-C) Levels of ubiquitinated Cav1.2 and AC6 in CUL4B overexpression and control TCP52 cells. HA-tagged ubiquitin was transiently transfected into WT and CUL4B overexpression TCP52 cell lines in the presence or absence of $10 \mathrm{nM}$ MG-132. The ubiquitinated proteins were pulled down with anti-HA beads, and ubiquitinated Cav1.2 and AC6 were detected with specific antibodies. Representative Western blots from at least 3 independent experiments are shown. (D-I) qChIP analysis of the recruitment of the indicated proteins on specific regions of the Cav1.2 promoter in CUL4B knockdown and control TCP52 cells. Whereas CUL4B, DDB1, $\mathrm{K} 119$-ubiquitinated-H2A, EZH2, and $\mathrm{K} 27-3$-methylated $\mathrm{H} 3$ protein bound to the -1500 to -1219 region of the Cav1.2 promoter, K4-3-methylated $\mathrm{H} 3$ bound to the -1060 to -900 region $(n=3)$. (J) qChIP analysis of the recruitment of CUL4B on specific regions of the Cav1.2 and AC6 promoter in HHEX knockdown and control TCP52 cells $(n=4)$. ( $(\mathbf{K}$ and $\mathbf{L})$ qChIP analysis of HHEX binding patterns of the Cav1.2 and AC6 promoters in TCP52 cells $(n=4)$. (M) Coimmunoprecipitation of HHEX and the CRL4B-PRC2 component in TCP52 cell lines. TCP52 cells were lysed and incubated with HHEX antibody or normal rabbit/mouse IgG on a rotator at $4^{\circ} \mathrm{C}$ overnight, followed by the addition of protein $A / C$ beads for 2 hours at $4^{\circ} \mathrm{C}$. Beads were then washed 5 times with lysis buffer. ${ }^{*} P<$ $0.05 ;{ }^{* *} P<0.01 ;{ }^{* *} P<0.001$. CUL4B knockdown cells were compared with control shRNA-treated cells. Bars represent mean \pm SEM. All data were analyzed using 1-way ANOVA.

cells, as demonstrated by coimmunoprecipitation assays (Figure $6 \mathrm{M}$ and Supplemental Figure 10D). Together, these results show that HHEX participates in the epigenetic regulation of Cav1.2 and AC6 by CUL4B in pancreatic $\delta$ cells.

Constitution of the CRL4B-PRC2 complex regulating Cav1.2 and AC6 expression. To further define the functional interplay between the CRL4B and PRC2 complexes in the transcriptional repression of Cav1.2 and AC6, we performed ChIP - Re-ChIP experiments. Soluble chromatin from TGP52 cells was first immunoprecipitated with antibodies against CUL4B, DDB1, or EZH2 and subsequently reimmunoprecipitated with the other antibodies. The results indicated that the Cav1.2 and AC6 promoters that immunoprecipitated with CUL4B antibody could be reimmunoprecipitated with antibodies against DDB1 and EZH2 (Figure 7, A and B). Similar results were acquired in sequential ChIP using DDB1 or EZH2 as the first antibody (Figure 7, A and B). These data support the notion that CUL4B, DDB1, and EZH2 constitute 1 multi-subunit complex at the promoters of targeted genes.

We next knocked down CUL4B, DDB1, and EZH2 individually to determine how the CRL4B-PRC2 complex is assembled at promoter regions of Cav1.2 and AC6. CUL4B and DDB1 knockdown drastically reduced the recruitment of other components of the CRL4B-PRC2 complex as well as H3K27me3 levels of promoter regions of Cav1.2 and AC6 (Figure 7, C and D, and Supplemental Figure 9, A and B). In contrast, knockdown of EZH2 did not affect the recruitment of CUL4B and DDB1 to the promoter region of Cav1.2 and AC6 (Figure 7, C and D, and Supplemental Figure 9, A and B). These data suggest that CRL4B promotes the recruitment and/or retention of PRC2 at the promoter regions of Cav1.2 and AC6. Critically, when we knocked down EZH2 or DDB1 in TGP52 cells, we observed significant increases in protein levels of Cav1.2 and AC6 (Figure 7, E and F, and Supplemental Figure 9, C and D). Moreover, high glucose-induced somatostatin secretion levels were significantly increased in EZH2 or
DDB1 knockdown TGP52 cells and to an extent similar to that observed in CUL4B knockdown cells (Figure 7G). Together, these data demonstrate that the integrity of the CRL4B-PRC2 complex is required for the transcriptional repression of Cav1.2 and AC6 in pancreatic $\delta$ cells (Figure $7 \mathrm{H}$ ).

Glucose and UCN3 inversely regulate Cav1.2 and AC6 expression by modulating the CRL $4 B-P R C 2$ complex. We monitored how protein levels of key second messenger regulators including Cav1.2 and AC6 are regulated by the CRL4B-PRC2 complex in TGP52 cells in response to different stimulations (e.g., glucose stimulation or UCN3 action). Interestingly, a significant increase in CUL4B protein levels was observed in high glucose-treated islets and TGP52 cells in a time-dependent manner (Figure 8, A and B, and Supplemental Figure 11, A and B). Similarly, EZH2 protein levels increased after persistently high glucose application whereas protein levels of Cav1.2 and AC6 decreased (Figure 8, C and D). In contrast, UCN3 induced significant decreases in the protein levels of CUL4B and EZH2 in TGP52 cells whereas increases in Cav1.2 and AC6 expression were observed with continued incubation (Figure 8, E and F). A similar inverse trend in Cul4b, Cav1.2, and AC6 mRNA levels was observed in TGP52 cells and primary pancreatic $\delta$ cells (Figure 8, G and $\mathrm{H}$, and Supplemental Figure 11, C-E). Importantly, CUL4B knockdown and Ast2B application in TGP52 cells eliminated protein- and mRNA-level changes in Cav1.2 and AC6 in response to high glucose levels and UCN3 stimulation, respectively (Figure 8, I-L, and Supplemental Figure 11F). These data showed that CRHR2 activity and CUL4B may regulate Cav1.2 and AC6 expression in pancreatic $\delta$ cells in response to high glucose or UCN3 levels and thus may play a role in the regulation of somatostatin secretion.

We therefore activated CRHR2 in human pancreatic islets via incubation with UCN3 for 4 hours. Previous studies have demonstrated that UCN3 potentiates high glucose-induced somatostatin secretion, which was inhibited by the L-type calcium channel blocker isradipine (5). Confirming previous findings, we observed that 4 hours of incubation with UCN3 caused an adaptive increase in somatostatin secretion in human islets in response to high glucose, which was eliminated by incubation with the AC inhibitor DDA or the Cav1.2 antagonist isradipine (Figure 8M). Moreover, when we used selective NEDD8activating enzyme (NAE) inhibitors (MLN4924) to block the CRL4B function in human islets, significantly higher levels of somatostatin secretion in response to high glucose levels were observed (Figure $8 \mathrm{~N}$ ). The effects of MLN4924 on human islet $\delta$ cells were eliminated through the incubation of either the AC inhibitor DDA or the Cav1.2 antagonist isradipine (Figure 8N). Therefore, the epigenetic mechanism modulates the extent of glucose-induced somatostatin secretion, which requires both $\mathrm{AC}$ and L-type calcium channel activities.

\section{Discussion}

The pancreatic islets of Langerhans are composed of at least 5 different cell types, including insulin-secreting $\beta$ cells, glucagonsecreting $\alpha$ cells, somatostatin-secreting $\delta$ cells, ghrelin-secreting $\varepsilon$ cells, and pancreatic polypeptide-secreting pp cells (44). Specific paracrine interactions among these islet cells are critical in mediating appropriate responses to different physiological contexts that 
A

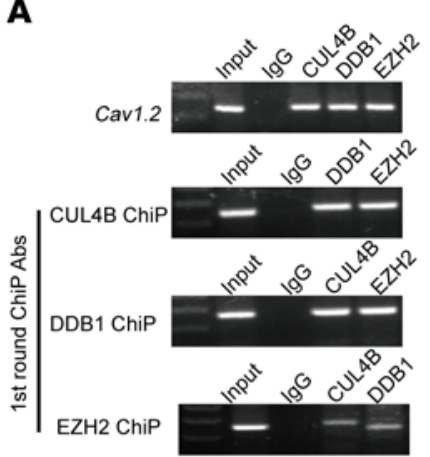

B

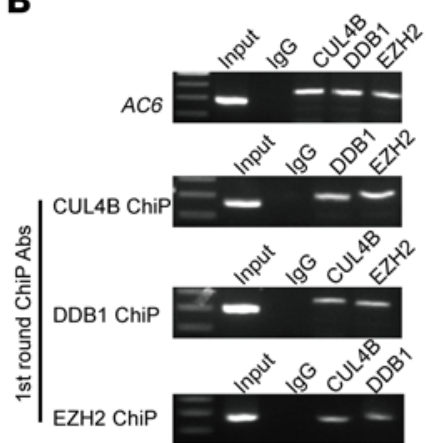

C

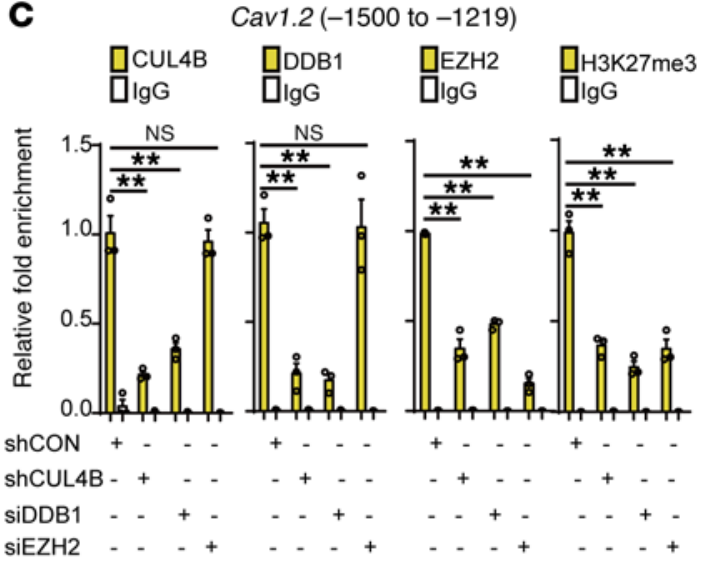

D
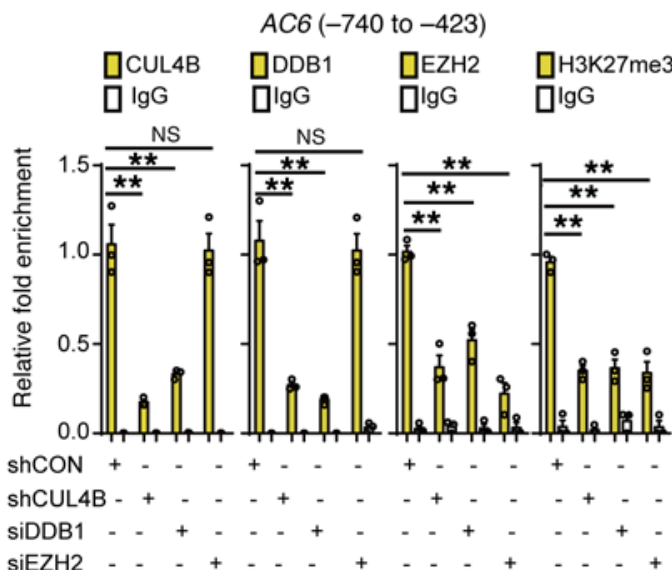

E

IB:DDB1

IB:Cav1.2

IB:AC6

IB:GAPDH
$\mathbf{F}$

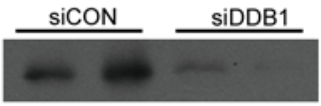

IB:EZH2

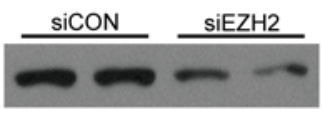

IB:Cav1.2

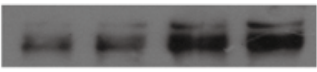

IB:AC6

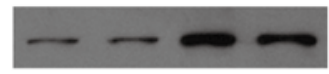

IB:GAPDH

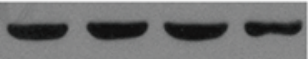

G

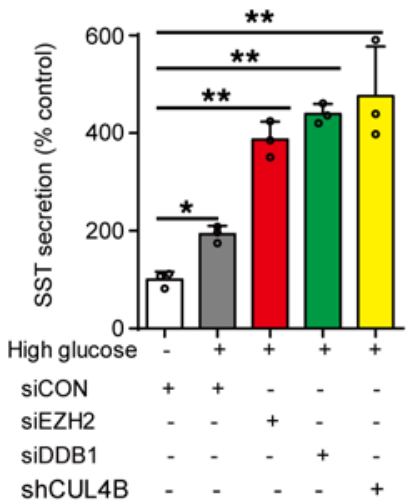

H

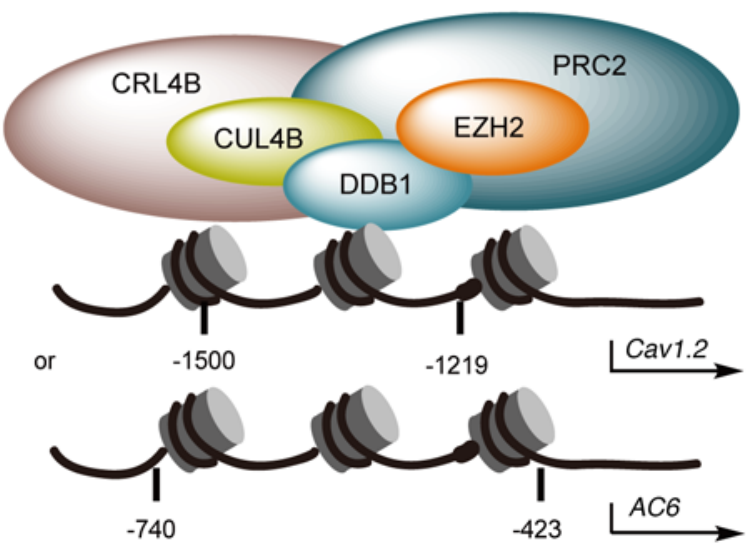

Figure 7. CUL4B and DDB1 regulate somatostatin secretion by recruiting EZH2 to constitute a functional CRL4B-PRC2 complex at the promoter regions of Cav1.2 and AC6. (A and B) ChIP and Re-ChIP (sequential ChIP) experiments revealed that CUL4B, DDB1, and EZH2 form multiple protein complexes in the promoter regions of Cav1.2 (A) and AC6 (B) in TGP52 pancreatic $\delta$ cells. (C and $\mathbf{D})$ qChIP analysis of the Cav1.2 (C) and AC6 (D) promoters in CUL4B, DDB1, or EZH2 knockdown TCP52 cells using the indicated antibodies. The results indicate that CRL4B promotes the recruitment of PRC2 to the promoters of Cav1.2 and AC6 $(n=3)$. (E and $\mathbf{F})$ Western blot analysis demonstrated increased expression of Cav1.2 and AC6 in DDB1 (E) and EZH2 (F) knockdown TCP52 cells. A representative Western blot from at least 3 experiments is shown. (C) High glucose-induced somatostatin secretion in CUL4B, DDB1, and EZH2 knockdown TCP52 cells after 15 minutes of stimulation $(n=3)$. (H) Schematic of the sequential IP strategy and a model of CUL4B/DDB1/EZH2 assembly at the promoters of Cav1.2 and A6. ${ }^{*} P<0.05 ;{ }^{* *} P<0.01$. CUL4B, DDB1, and EZH2 knockdown cells were compared with control shRNA- or siRNA-treated cells. Bars represent mean \pm SEM. All data were analyzed using 1-way ANOVA.

cannot be achieved by a single $\beta$ or $\alpha$ cell $(2,4,5,45)$. For example, recent studies have shown that the loss of UCN3 stimulation by pancreatic $\beta$ cells decreases somatostatin-dependent negative feedback and disrupts glucose homeostasis (5). As an important paracrine factor, UCN3 potentiates high glucose-induced somatostatin secretion, which requires L-type calcium channel activity (5). Here, we confirmed these observations in human islets (Figure
$8 \mathrm{M})$. Moreover, our results demonstrate that somatostatin secretion is controlled by an epigenetic mechanism through modulation of the expression of CUL4B and EZH2, 2 core components of the CRL4B-PRC2 supercomplex. High concentrations of glucose induce somatostatin secretion, and this is immediately followed by gradually increasing levels of CUL4B and EZH2 expression (Figure 8, A, B, and G, and Figure 9). When high glucose levels per- 
A

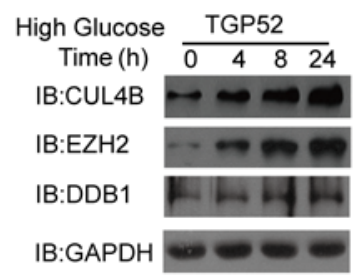

C

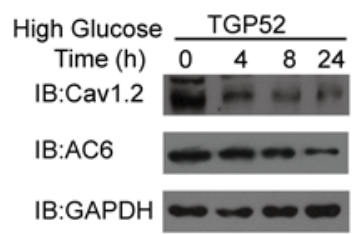

I

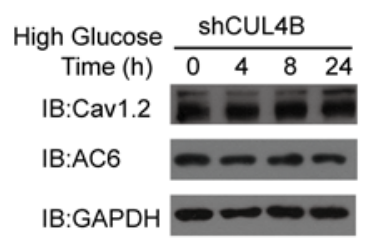

B

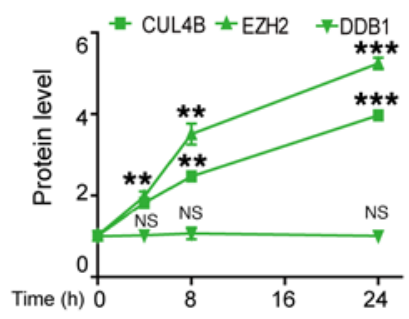

D

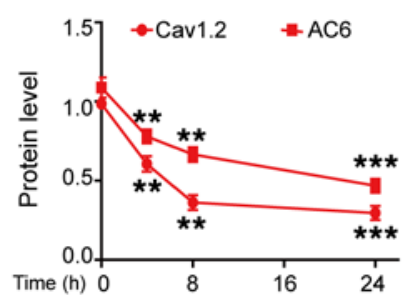

J

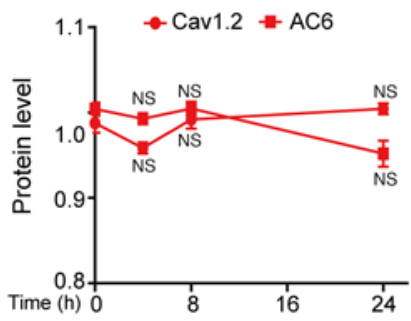

E UCN3 (100 nM) TGP52

Time (h) $\begin{array}{llll}0 & 4 & 8 & 24\end{array}$

IB:CUL $4 B$

IB:EZH2

IB:DDB1

IB:Cav1.2

IB:AC6

IB:GAPDH

G

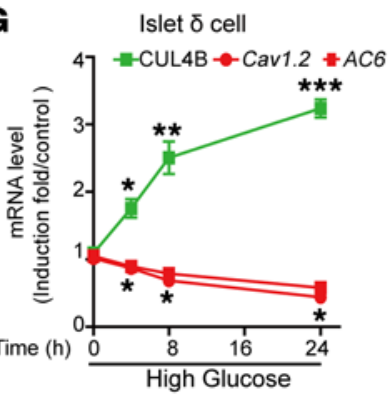

K

UCN3 (100 nM)

Astressin 2B (100 nM)

\begin{tabular}{lllll}
\cline { 2 - 4 } Time (h) & 0 & 4 & 8 & 24
\end{tabular}

IB:CUL4B

IB:EZH2

IB:DDB1

IB:Cav1.2

IB:AC6
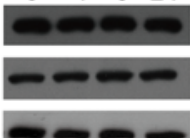

Etrat

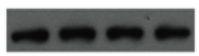

IB:GAPDH

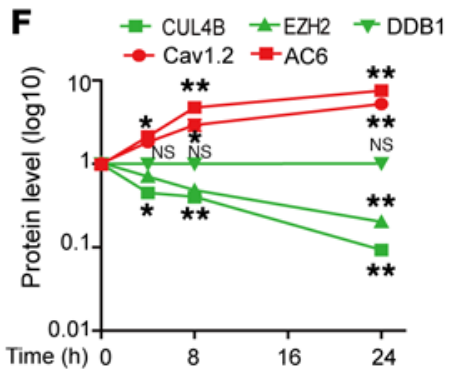

H

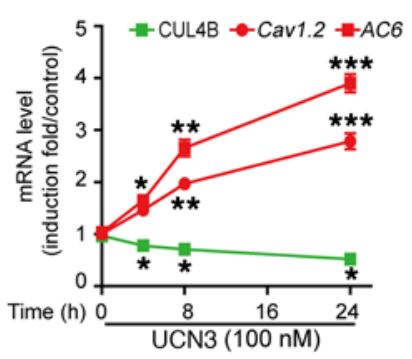

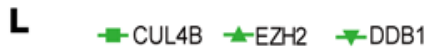

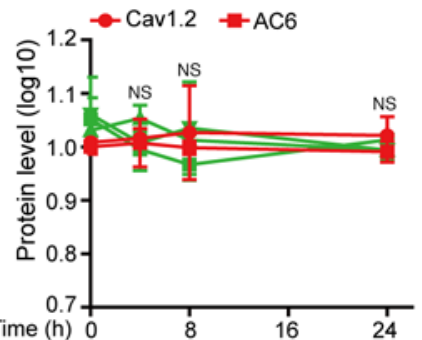

M

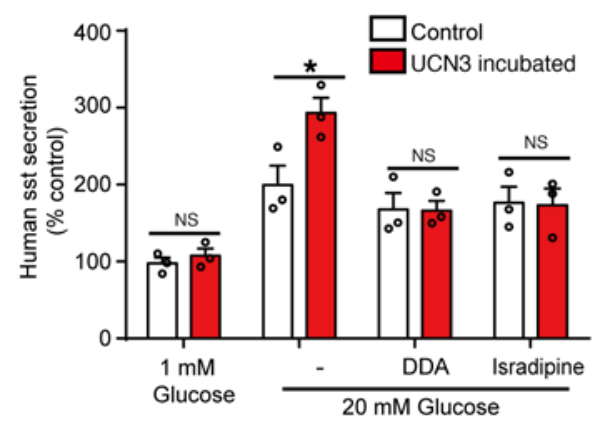

$\mathbf{N}$

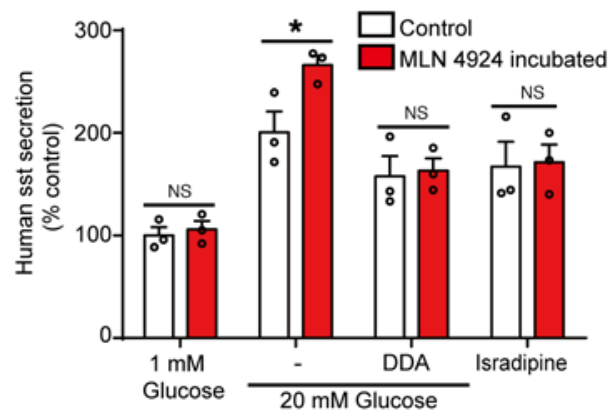

Figure 8. Reciprocal regulation of the CUL4B/PRC2 complex, Cav1.2, and AC6 by glucose and UCN3 in pancreatic $\delta$ cells. (A and B) Protein-level changes in CUL4B, EZH2, and DDB1 expression in TCP52 cells stimulated with $20 \mathrm{mM}$ glucose for the indicated time intervals. (C, D, I, and J) Protein-level changes in Cav1.2 and AC6 expression in CUL4B knockdown and control TCP52 cells stimulated with $20 \mathrm{mM}$ glucose for the indicated time intervals. (E and F) Protein level changes in CUL4B, EZH2, DDB1, Cav1.2, and AC6 expression in TCP52 cells stimulated with 100 nM UCN3. (G and $\mathbf{H})$. The mRNA levels of Cul4b, Cav1.2, and AC6 in primary isolated islet $\delta$ cells treated with $20 \mathrm{mM}$ glucose (G) or $100 \mathrm{nM} \mathrm{UCN3} \mathrm{(H)} \mathrm{for} \mathrm{the} \mathrm{indicated} \mathrm{number} \mathrm{of} \mathrm{hours.} \mathrm{(K} \mathrm{and} \mathbf{L})$ Protein level changes in CUL4B, EZH2, DDB1, Cav1.2, and AC6 expression in TCP52 cells stimulated with $100 \mathrm{nM}$ UCN3 and CRHR2-selective antagonist Ast2B for the indicated time intervals. (A, C, E, I, and $\mathbf{K}$ ) Representative Western blots from at least 3 independent experiments are shown. (B, D, F, J, and L) Statistical analyses of A, C, E, I and $\mathbf{K}$, respectively. (M and $\mathbf{N})$ AC inhibitor DDA $(100 \mu \mathrm{M})$ and L-type channel blocker isradipine $(10 \mu \mathrm{M})$ completely blocked the enhanced somatostatin secretion from human islets incubated with UCN3 (100 nM) (M) or MLN4924 (200 nM) (N) compared with their control islets. ${ }^{*} P<$ $0.05 ;{ }^{* *} P<0.01 ;{ }^{* *} P<0.001$. Glucose- and UCN3-stimulated cells were compared with nonstimulated cells, and human islets incubated with UCN3 were compared with control islets. Bars represent mean \pm SEM. All data were analyzed through 1-way ANOVA.

sist, these signals may progressively decrease somatostatin secretion and impede the somatostatin negative feedback loop. In contrast, UCN3 inhibits the expression of CUL4B and EZH2, thereby increasing somatostatin secretion in a time-dependent manner and ensuring decreased insulin release after glucose levels return to baseline levels (Figure 8, E, F, and H, and Figure 9). Therefore, we found that the CRL4B-PRC2 complex plays an important role in the modulation of paracrine interactions between pancreatic $\delta$ 


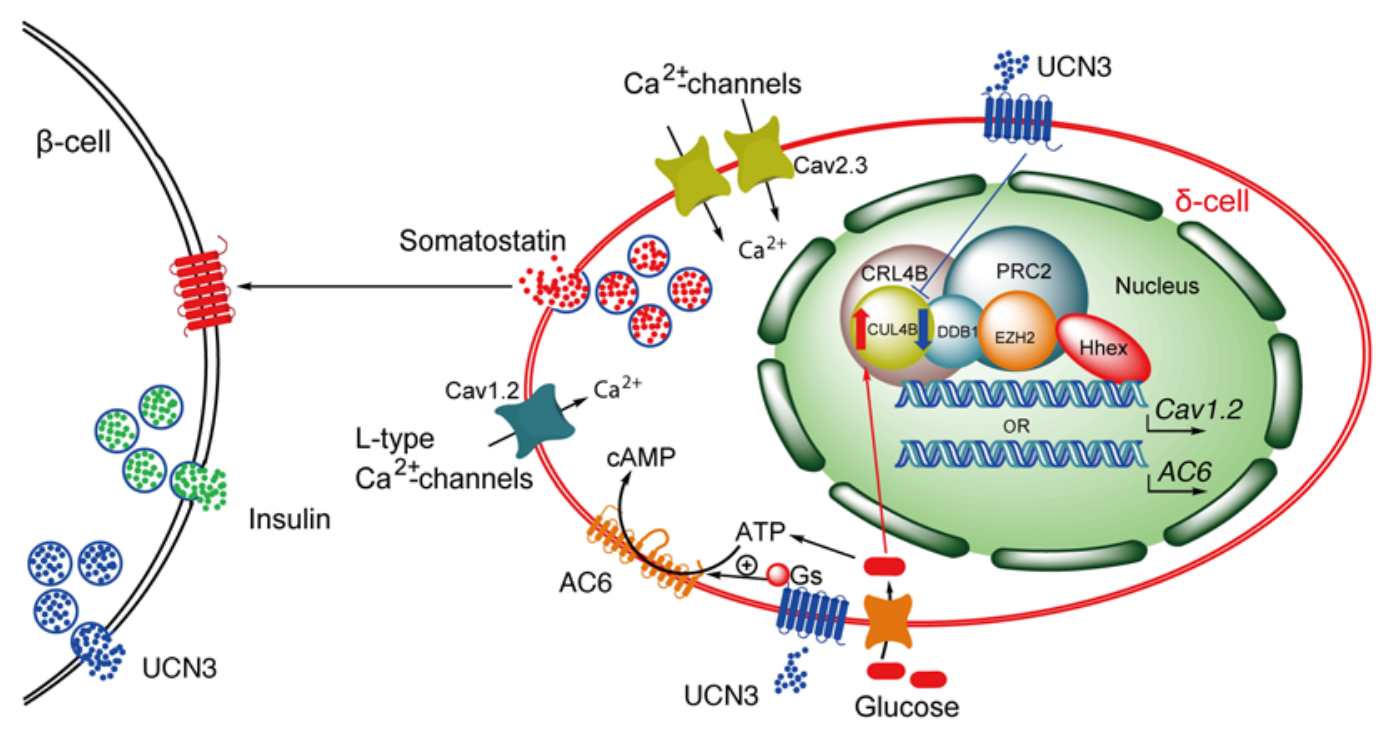

Figure 9. Graphic model of the CRL4B-PRC2 complex-mediated regulation of pancreatic somatostatin paracrine signaling and of adjustments in glucose homeostasis in response to high glucose or UCN3 stimulation. As demonstrated by previous studies, paracrine factor UCN3 and insulin were coreleased from the same vesicles from pancreatic $\beta$ cells (5). The secreted UCN3 increases somatostatin secretion in response to high glucose stimulation in pancreatic islets, and L-type channel activity is required for the potentiation effects of UCN3 (5). Here, we found that in response to sustained UCN3 stimulation, the expression levels of CUL4B and EZH2, the core components of CRL4B-PRC2, decreased in pancreatic $\delta$ cells. Under the control of HHEX, the CRL4B-PRC2 complex epigenetically regulates the expression of calcium channel Cav1.2 and adenylyl cyclase AC6 through the modulation of methylation and ubiquitination states of the histone proteins that bind to the promoters of these 2 proteins in pancreatic $\delta$ cells. The decreased expression of CUL4B and EZH2 releases the repressive role of the CRL4B-PRC2 complex in the expression of Cav1.2 and AC6, thereby increasing levels for second messengers $\mathrm{Ca}^{2+}$ and cAMP in pancreatic $\delta$ cells and ultimately leading to increased somatostatin secretion levels. Consistent with a previous study (5), this feedback loop is essential for avoiding excessive sustained insulin secretion in a timely manner and for coordinating normal glucose homeostasis. In contrast, the protein levels of CUL4B and EZH2 increase in response to sustained high glucose stimulation. The upregulated CRL4B-PRC2 complex function decreases the expression of Cav1.2 and AC6, which in turn decreases somatostatin secretion and thus ensures sufficient insulin secretion for maintaining glucose homeostasis. The epigenetic regulation of somatostatin secretion by the CRL4B-PRC2 complex is important for maintaining glucose homeostasis.

and $\beta$ cells. Moreover, we identified that CUL4B dysfunction in pancreatic $\delta$ cells results in insufficient insulin secretion and in glucose homeostasis disturbance.

Recent studies have demonstrated that both the R-type calcium channel and the L-type calcium channel play important roles in the modulation of somatostatin secretion $(5,12)$. Here, we demonstrate that the expression levels of L-type calcium channels rather than R-type calcium channels are closely regulated by the CRL4B-PRC2 complex. Cav1.2 mediates excitation-contraction coupling in the heart (43), excitation-transcription coupling in the brain, and excitation-secretion coupling in many endocrine cells (46). Mutations in the Cav1.2 gene cause psychiatric disorders, such as bipolar disorder and schizophrenia (47). However, little is known of the transcriptional regulation of Cav1.2 $(48,49)$, and specific epigenetic mechanisms that regulate Cav1.2 have not been identified. Here, we found that CUL4B epigenetically modifies Cav1.2 expression levels in the CRL4B-PRC2 complex. Our data demonstrate that the CRL4B-PRC2 complex binds to the Cav1.2 promoter, thus repressing its expression by catalyzing H2K119 monoubiquitin and H3K27 methylation (Figure 6, D-I; Supplemental Figure 8, E-J, and Figure 9). Moreover, we observed that Cav1.2 expression was specifically regulated in pancreatic $\delta$ cells, but not in pancreatic $\beta$ cells, owing to selective HHEX interactions (Figure 6, J-M). The diabetes gene Hhex is specifically expressed in pancreatic $\delta$ cells and is important for $\delta$ cell differentiation (11). In TGP52 cells, HHEX associates with the CRL4B-PRC2 complex to control the expression of Cav1.2 and AC6 (Figure 6, J-M). These results further indicate that the epigenetic control of key regulators of hormone secretion is cell-type specific.

Notably, Ins2-Cre and Sst-Cre mice may exhibit induced recombination in nonislet cells, such as in the brain and in the stomach $(50,51)$. Although we demonstrated that the hyposecretion of insulin and the hypersecretion of somatostatin from pancreatic islets aid in maintaining normal glucose homeostasis (Figure $1 \mathrm{H}$ and Figure 2), the deficiencies of CUL4B in SST-synthesized cells other than islets may also contribute to impaired glucose tolerance in $\mathrm{Sst}-\mathrm{Cre}^{+/-} \mathrm{Cul}_{4} b^{A / Y}$ mice, a possibility that requires further study. In addition, many of our biochemical studies used the pancreatic epithelial-like TGP52 cell line, an imperfect model for monitoring epigenetic interactions of islets. Therefore, in vivo chromatin characterizations and their relationships to the CRL4B-PRC2 complex in islets may provide important information on the epigenetic regulation of islet paracrine interactions. In the current study, to further extend the identified functional importance of the epigenetic mechanism for the regulation of somatostatin secretion in human islets, we blocked CUL4B activity using the NEDD8activating enzyme inhibitor MLN4924 (Figure 8N), which adaptively potentiates high glucose-induced somatostatin secretion, phenocopying the effects of UCN3 (Figure 8M), as shown in previously published results (5). Moreover, DDA and isradipine application eliminated the effects of long-term MLN4924 treatment on facilitating somatostatin secretion, suggesting that our hypothesis 
derived from mouse islets and TGP52 cells may also be applied to human tissues (Figure 8N). Therefore, the dysfunction of epigenetic regulation in pancreatic $\delta$ cells may lead to somatostatin hypersecretion. Given that antagonizing CRHR2 activity rescued somatostatin hypersecretion in a mouse model of Cul4b deficiency specifically in $\delta$ cells (Figure $4, C$ and D) and that CUL $4 B$ mutations are related to obesity (24), a CRHR2 antagonist, such as Ast2B (5), might be developed as a more specific therapeutic method for malfunctions of CRL4B-PRC2 complexes.

Together, our results reveal an important epigenetic mechanism that modulates paracrine interactions between pancreatic $\delta$ and $\beta$ cells during the maintenance of glucose homeostasis, the functional importance of the CRL4B-PRC2 complex in this system of regulation, and the mechanism by which the expression of a calcium channel and adenylyl cyclase are specifically controlled in pancreatic $\delta$ cells.

\section{Methods}

Animals. The cre driver mice Sst-Cre (Ssttm 2.1[cre], stock number 013044) and Ins2-Cre (B6.Cg-Tg[Ins2-cre]25Mgn/J, stock number 003573) and GFP mice (stock number 007676) were purchased from Jackson Laboratory. Ssttm 2.1 (cre) mice with a congenic C57BL/6 background were obtained by crossing Ssttm 2.1 (cre) mice with a C57BL/6 background. Cul4b $b^{\sharp / h}$ mice were generated at the National Resource Center of Mutant Mice/Model Animal Research Center of Nanjing University, Nanjing, Jiangsu, China) (52). Tdt-tomato mice were obtained from Tian Xue (53). All experiments were performed with male mice.

Glucose tolerance test. Male mice 12 to 14 weeks of age were fasted for 16 hours and given free access to water. Blood glucose levels were measured in tail blood by using a FreeStyle Lite Glucose Meter (Roche) before (baseline, 0 minutes) as well as 15, 30, 60, 90, and 120 minutes after mice were intraperitoneally challenged with $2 \mathrm{~g} / \mathrm{kg}$ body weight glucose.

ChIP. We analyzed the enrichment of the DNA template by conventional PCR using primers specific for Cav1.1, Cav1.2, Cav1.3, Cav1.4, $A C 6$, or $A C 7$ promoters. All primers used for ChIP assay of CRL4B or PRC2 complexes are listed in Supplemental Table 1. Detailed information is available in Supplemental Materials and Methods.

RNA extraction and quantitative reverse-transcriptase PCR. RNA from CUL4B knockdown and control TGP52 cells as well as islets derived from $\mathrm{Sst}-\mathrm{Cre}^{+/-} \mathrm{GFP} \mathrm{P}^{\mathrm{fl}+} \mathrm{Cul}^{\mathrm{C}} \mathrm{b}^{A / \mathrm{Y}}$ mice and their WT littermates (Sst-Cre ${ }^{+/-} \mathrm{GFP}^{\mathrm{fl} /+} \mathrm{Cul}^{+} \mathrm{b}^{+/ Y}$ mice) were extracted with TRIzol reagent (Invitrogen). The single $\delta$ cell was picked by hand and incubated with buffer containing the RNase inhibitor (N8080119, Thermo
Fisher Scientific). We performed cDNA synthesis using the qRT-PCR Kit (Toyobo, FSQ-101). We conducted quantitative reverse-transcriptase PCR (qRT-PCR) in the LightCycler qPCR apparatus (Bio-Rad) with the FastStart SYBR Green Master (Roche). All primers used for qRT-PCR assay are listed in Supplemental Table 2.

Statistics. All data are presented as mean $\pm \mathrm{SD}$ or mean \pm SEM. Differences between different groups were determined using 1-way ANOVA in GraphPad Prism 5 software. A P value of less than 0.05 was considered significant.

Study approval. The use of isolated human islets was approved by the ethics committee of The Central South University Second Xiangya Hospital (protocol MSRC2016LF). All animal care and experiments were reviewed and approved by the Animal Use Committee of Shandong University School of Medicine.

\section{Author contributions}

$\mathrm{XY}$ and YG conceived and initiated the project. QL, XY, and JPS designed all of the experiments. JPS, XY, and YG supervised the overall project design and execution. QL, MC, F Yang, NL, XY, and JPS participated in data analysis and interpretation. QL, NL, ZY, YW, XZ, SLN, SW, and HQ performed mouse experiments. J Zhang, FL, J Zhao, and LG provided human islets. QL, NL, DZ, and HS performed cell biology. BJ, HH, PSL, DH, AL, F Yi, and TX provided insightful ideas and contributed to experimental design. $\mathrm{XY}$ and JPS wrote the manuscript. All of the authors have seen and commented on the manuscript.

\section{Acknowledgments}

We acknowledge support from the National Key Basic Research Program of China (2013CB967700 to XY and TX;2014CB910500 to FL), the National Basic Research Program of China (2014CB910500 to FL), the National Natural Science Foundation of China (31471102, 31671197 to XY; 31470789 to JPS; 81330050 to YG; 81370017 to J Zhang; 31322024 to TX), the Shandong Natural Science Fund for Distinguished Young Scholars (JQ201320 to XY and JQ201517 to JPS), the Natural Science Foundation of Shandong Province (ZR2016HZ01 to YG), the Fundamental Research Fund of Shandong University (2014JC029 to XY), and the Program for Changjiang Scholars and Innovative Research Team in University (IRT13028).

Address correspondence to: Xiao Yu or Yoaqin Gong, Shandong University School of Medicine, Jinan, Shandong, China. Phone: 86.531.88381910; Email: yuxiao@sdu.edu.cn (X. Yu).Phone: 86.531.88382115; Email: yxg8@sdu.edu.cn (Y. Gong).
1. Molina J, Rodriguez-Diaz R, Fachado A, Jacques-Silva MC, Berggren PO, Caicedo A. Control of insulin secretion by cholinergic signaling in the human pancreatic islet. Diabetes. 2014;63(8):2714-2726.

2. Unger RH, Orci L. Paracrinology of islets and the paracrinopathy of diabetes. Proc Natl Acad SciUS A. 2010;107(37):16009-16012.

3. Huising MO. Tuning to the right signal. Diabetologia. 2015;58(6):1146-1148.

4. Caicedo A. Paracrine and autocrine interactions in the human islet: more than meets the eye.
Semin Cell Dev Biol. 2013;24(1):11-21.

5. van der Meulen T, et al. Urocortin3 mediates somatostatin-dependent negative feedback control of insulin secretion. Nat Med. 2015;21(7):769-776.

6. Kusminski CM, et al. MitoNEET-Parkin Effects in Pancreatic $\alpha$ - and $\beta$-Cells, Cellular Survival, and Intrainsular Cross Talk. Diabetes. 2016;65(6):1534-1555.

7. Wang MY, et al. Glucagon receptor antibody completely suppresses type 1 diabetes phenotype without insulin by disrupting a novel diabetogenic pathway. Proc Natl Acad Sci US A.
2015;112(8):2503-2508.

8. Segerstolpe Å, et al. Single-cell transcriptome profiling of human pancreatic islets in health and type 2 diabetes. Cell Metab. 2016;24(4):593-607.

9. Xin Y, et al. RNA sequencing of single human islet cells reveals type 2 diabetes genes. Cell Metab. 2016;24(4):608-615.

10. Hauge-Evans AC, et al. Somatostatin secreted by islet delta-cells fulfills multiple roles as a paracrine regulator of islet function. Diabetes. 2009;58(2):403-411.

11. Zhang J, McKenna LB, Bogue CW, Kaestner 
KH. The diabetes gene Hhex maintains $\delta$-cel differentiation and islet function. Genes Dev. 2014;28(8):829-834.

12. Zhang Q, et al. R-type $\mathrm{Ca}(2+)$-channel-evoked CICR regulates glucose-induced somatostatin secretion. Nat Cell Biol. 2007;9(4):453-460.

13. Wang HM, et al. A stress response pathway in mice upregulates somatostatin level and transcription in pancreatic delta cells through Gs and $\beta$-arrestin 1. Diabetologia. 2014;57(9):1899-1910.

14. Claiborn KC, Sachdeva MM, Cannon CE, Groff DN, Singer JD, Stoffers DA. Pcif1 modulates $\mathrm{Pdx} 1$ protein stability and pancreatic $\beta$ cell function and survival in mice. J Clin Invest. 2010;120(10):3713-3721.

15. Zhong L, Georgia S, Tschen SI, Nakayama K, Nakayama K, Bhushan A. Essential role of Skp2-mediated p27 degradation in growth and adaptive expansion of pancreatic beta cells. J Clin Invest. 2007;117(10):2869-2876.

16. Sancho R, Gruber R, Gu G, Behrens A. Loss of Fbw7 reprograms adult pancreatic ductal cells into $\alpha, \delta$, and $\beta$ cells. Cell Stem Cell. 2014;15(2):139-153.

17. Hoeck JD, et al. Fbw7 controls neural stem cell differentiation and progenitor apoptosis via Notch and c-Jun. Nat Neurosci. 2010;13(11):1365-1372.

18. Groh BS, Yan F, Smith MD, Yu Y, Chen X, Xiong $Y$. The antiobesity factor WDTC1 suppresses adipogenesis via the CRL4WDTC1 E3 ligase. EMBO Rep. 2016;17(5):638-647.

19. Lin GN, et al. Spatiotemporal 16p11.2 protein network implicates cortical late mid-fetal brain development and KCTD13-Cul3-RhoA pathway in psychiatric diseases. Neuron. 2015;85(4):742-754.

20. Schumacher FR, et al. Characterisation of the Cullin-3 mutation that causes a severe form of familial hypertension and hyperkalaemia. $E M B O$ Mol Med. 2015;7(10):1285-1306.

21. Yoshizawa T, et al. SIRT7 controls hepatic lipid metabolism by regulating the ubiquitin-proteasome pathway. Cell Metab. 2014;19(4):712-721.

22. Yu C, et al. CRL4 complex regulates mammalian oocyte survival and reprogramming by activation of TET proteins. Science. 2013;342(6165):1518-1521.

23. Zou Y, et al. Mutation in CUL4B, which encodes a member of cullin-RING ubiquitin ligase complex, causes X-linked mental retardation. Am J Hum Genet. 2007;80(3):561-566.

24. Tarpey PS, et al. Mutations in CUL4B, which encodes a ubiquitin E3 ligase subunit, cause an $\mathrm{X}$-linked mental retardation syndrome associated with aggressive outbursts, seizures, relative macrocephaly, central obesity, hypogonadism, pes cavus, and tremor. Am J Hum Genet. 2007;80(2):345-352.

25. Zha Z, et al. A non-canonical function of $G \beta$ as a subunit of E3 ligase in targeting GRK2 ubiquitylation. Mol Cell. 2015;58(5):794-803.

26. Han J, Zhang H, Zhang H, Wang Z, Zhou H, Zhang Z. A Cul4 E3 ubiquitin ligase regulates histone hand-off during nucleosome assembly. Cell. 2013;155(4):817-829.

27. Liu L, et al. Essential role of the CUL4B ubiquitin ligase in extra-embryonic tissue development during mouse embryogenesis. Cell Res. 2012;22(8):1258-1269.

28. $\mathrm{Hu} \mathrm{H}$, et al. CRL4B catalyzes H2AK119 monoubiquitination and coordinates with PRC2 to promote tumorigenesis. Cancer Cell. 2012;22(6):781-795.

29. Yang Y, et al. CRL4B promotes tumorigenesis by coordinating with SUV39H1/HP1/DNMT3A in DNA methylation-based epigenetic silencing. Oncogene. 2015;34(1):104-118.

30. Nakagawa T, Xiong Y. X-linked mental retardation gene CUL4B targets ubiquitylation of H3K4 methyltransferase component WDR5 and regulates neuronal gene expression. Mol Cell. 2011;43(3):381-391.

31. Li P, et al. Lack of CUL4B in adipocytes promotes PPAR $\gamma$-mediated adipose tissue expansion and insulin sensitivity. Diabetes. 2017;66(2):300-313.

32. Kumar U, et al. Subtype-selective expression of the five somatostatin receptors (hSSTR1-5) in human pancreatic islet cells: a quantitative double-label immunohistochemical analysis. Diabetes. 1999;48(1):77-85.

33. Braun M. The somatostatin receptor in human pancreatic $\beta$-cells. Vitam Horm. 2014;95:165-193.

34. Yue JT, Burdett E, Coy DH, Giacca A, Efendic S, Vranic M. Somatostatin receptor type 2 antagonism improves glucagon and corticosterone counterregulatory responses to hypoglycemia in streptozotocin-induced diabetic rats. Diabetes. 2012;61(1):197-207.

35. Rocheville M, Lange DC, Kumar U, Patel SC, Patel RC, Patel YC. Receptors for dopamine and somatostatin: formation of hetero-oligomers with enhanced functional activity. Science. 2000;288(5463):154-157.

36. Kelly C, Flatt PR, McClenaghan NH. Cell-to-cell communication and cellular environment alter the somatostatin status of delta cells. Biochem Biophys Res Commun. 2010;399(2):162-166.

37. Gregg T, et al. Pancreatic $\beta$-cells from mice offset age-associated mitochondrial deficiency with reduced KATP channel activity. Diabetes. 2016;65(9):2700-2710.

38. Ashcroft FM, Rorsman P. K (ATP) channels and islet hormone secretion: new insights and controversies. Nat Rev Endocrinol. 2013;9(11):660-669.

39. Ning SL, et al. Different downstream signalling of CCK1 receptors regulates distinct functions of CCK in pancreatic beta cells. Br J Pharmacol.
2015;172(21):5050-5067.

40. Dou HQ, et al. Thiopental-induced insulin secretion via activation of IP3-sensitive calcium stores in rat pancreatic $\beta$-cells. Am J Physiol Cell Physiol. 2012;302(5):C796-C803.

41. Dou H, et al. Calcium influx activates adenylyl cyclase 8 for sustained insulin secretion in rat pancreatic beta cells. Diabetologia. 2015;58(2):324-333.

42. Del Prete D, Rice RC, Rajadhyaksha AM, D'Adamio L. amyloid precursor protein (APP) may act as a substrate and a recognition unit for CRL4CRBN and Stub1 E3 ligases facilitating ubiquitination of proteins involved in presynaptic functions and neurodegeneration. J Biol Chem. 2016;291(33):17209-17227.

43. Hofmann F, Flockerzi V, Kahl S, Wegener JW. L-type CaV1.2 calcium channels: from in vitro findings to in vivo function. Physiol Rev. 2014;94(1):303-326.

44. Collombat $\mathrm{P}$, et al. The ectopic expression of Pax4 in the mouse pancreas converts progenitor cells into alpha and subsequently beta cells. Cell. 2009;138(3):449-462.

45. Chera S, et al. Diabetes recovery by age-dependent conversion of pancreatic $\delta$-cells into insulin producers. Nature. 2014;514(7523):503-507.

46. Groth RD, Tirko NN, Tsien RW. CaV1.2 calcium channels: just cut out to be regulated? Neuron. 2014;82(5):939-940.

47. Psychiatric GWAS Consortium Bipolar Disorder Working Group. Large-scale genome-wide association analysis of bipolar disorder identifies a new susceptibility locus near ODZ4. Nat Genet. 2011;43(10):977-983.

48. Shi XZ, Pazdrak K, Saada N, Dai B, Palade P, Sarna SK. Negative transcriptional regulation of human colonic smooth muscle Cav1.2 channels by $\mathrm{p} 50$ and p 65 subunits of nuclear factor-kappaB. Gastroenterology. 2005;129(5):1518-1532.

49. Rau F, et al. Misregulation of miR-1 processing is associated with heart defects in myotonic dystrophy. Nat Struct Mol Biol. 2011;18(7):840-845.

50. Mehran AE, et al. Hyperinsulinemia drives dietinduced obesity independently of brain insulin production. Cell Metab. 2012;16(6):723-737.

51. Egerod KL, et al. Transcriptional and functional characterization of the g protein-coupled receptor repertoire of gastric somatostatin cells. Endocrinology. 2015;156(11):3909-3923.

52. Jiang B, et al. Lack of Cul4b, an E3 ubiquitin ligase component, leads to embryonic lethality and abnormal placental development. PLoS One. 2012;7(5):e37070.

53. Do MT, et al. Photon capture and signalling by melanopsin retinal ganglion cells. Nature. 2009;457(7227):281-287. 